\title{
Malliavin Calculus for the Stochastic 2D Navier-Stokes Equation
}

\author{
Jonathan C. Mattingly \\ School of Mathematics, Institute for Advanced Studies, Princeton N.J, 08540 USA and \\ Department of Mathematics, Duke University, Box 90320, Durham, NC 27708-0320 USA. \\ Email:jonm@math.duke.edu \\ AND \\ Étienne Pardoux \\ LATP/CMI, Université de Provence, 39 rue F. Joliot Curie, 13453 Marseille cedex 13, \\ France.Email: pardoux@cmi.univ-mrs.fr
}

\begin{abstract}
We consider the incompressible, two dimensional Navier Stokes equation with periodic boundary conditions under the effect of an additive, white in time, stochastic forcing. Under mild restrictions on the geometry of the scales forced, we show that any finite dimensional projection of the solution possesses a smooth, strictly positive density with respect to Lebesgue measure. In particular, our conditions are viscosity independent. We are mainly interested in forcing which excites a very small number of modes. All of the results rely on proving the nondegeneracy of the infinite dimensional Malliavin matrix. (c) 2005 Wiley Periodicals, Inc.
\end{abstract}

\section{Introduction}

We consider the movement of a two-dimensional, incompressible fluid with mean flow zero under periodic boundary conditions. We analyze the problem using the vorticity formulation of the following form

$$
\left\{\begin{array}{l}
\frac{\partial w}{\partial t}(t, x)+B(w, w)(t, x)=v \Delta w(t, x)+\frac{\partial W}{\partial t}(t, x) \\
w(0, x)=w_{0}(x),
\end{array}\right.
$$

where $x=\left(x_{1}, x_{2}\right) \in \mathbf{T}^{2}$, the two-dimensional torus $[0,2 \pi] \times[0,2 \pi], v>0$ is the viscosity constant, $\frac{\partial W}{\partial t}$ is a white-in-time stochastic forcing to be specified below, and

$$
B(w, \tilde{w})=\sum_{i=1}^{2} u_{i}(x) \frac{\partial \tilde{w}}{\partial x_{i}}(x)
$$


where $u=\mathscr{K}(w)$. Here $\mathscr{K}$ is the Biot-Savart integral operator which will be defined next. First we define a convenient basis in which we will perform all explicit calculations. Setting $\mathbb{Z}_{+}^{2}=\left\{\left(j_{1}, j_{2}\right) \in \mathbb{Z}^{2}: j_{2}>0\right\} \cup\left\{\left(j_{1}, j_{2}\right) \in \mathbb{Z}^{2}: j_{1}>0, j_{2}=0\right\}$, $\mathbb{Z}_{-}^{2}=-\mathbb{Z}_{+}^{2}$ and $\mathbb{Z}_{0}^{2}=\mathbb{Z}_{+}^{2} \cup \mathbb{Z}_{-}^{2}$, we define a real Fourier basis for functions on $\mathbf{T}^{2}$ with zero spatial mean by

$$
e_{k}(x)= \begin{cases}\sin (k \cdot x) & k \in \mathbb{Z}_{+}^{2} \\ \cos (k \cdot x) & k \in \mathbb{Z}_{-}^{2} .\end{cases}
$$

We write $w(t, x)=\sum_{k \in \mathbb{Z}_{0}^{2}} \alpha_{k}(t) e_{k}(x)$ for the expansion of the solution in this basis. With this notation in the two-dimensional periodic setting we have the expression

$$
\mathscr{K}(w)=\sum_{k \in \mathbb{Z}_{0}^{2}} \frac{k^{\perp}}{\|k\|^{2}} \alpha_{k} e_{-k},
$$

where $k^{\perp}=\left(-k_{2}, k_{1}\right)$ and $\|k\|^{2}=k_{1}^{2}+k_{2}^{2}$. See for example [MB02] for more details on the deterministic vorticity formulation in a periodic domain. We use the vorticity formulation for simplicity. All of our results can be translated into statements about the velocity formulation of the problem.

We take the forcing to be of the form

$$
W(t, x)=\sum_{k \in \mathscr{Z}_{*}} W_{k}(t) e_{k}(x)
$$

where $\mathscr{Z}_{*}$ is a finite subset of $\mathbb{Z}_{0}^{2}$ and $\left\{W_{k}: k \in \mathscr{Z}_{*}\right\}$ is a collection of mutually independent standard scalar Brownian Motions on a probability space $(\Omega, \mathscr{F}, \mathbb{P})$. The fact that we force a finite collection of Fourier modes becomes important starting in Section 3. Up until then the analysis applies to a force acting on any linearly independent collection of functions from $\mathbf{T}^{2}$ into $\mathbb{R}$ which have spatial mean zero. The collection could even be infinite with a mild summability assumption.

We assume that $w_{0} \in \mathbb{L}^{2}=\left\{w \in L^{2}\left(\mathbf{T}^{2}, \mathbb{R}\right): \int w d x=0\right\}$. We will use $\|\cdot\|$ to denote the norm on $\mathbb{L}^{2}$ and $\langle\cdot, \cdot\rangle$ to denote the innerproduct. We also define $\mathbb{H}^{s}=\left\{w \in H^{s}\left(\mathbf{T}^{2}, \mathbb{R}\right): \int w d x=0\right\}$. Under these assumptions, it is standard that $w \in C\left([0,+\infty) ; \mathbb{L}^{2}\right) \cap L_{l o c}^{2}\left((0,+\infty) ; \mathbb{H}^{1}\right)$ [Fla94, DPZ96 MR04]. We will denote by $\|\cdot\|_{s}$ the natural norm on $\mathbb{H}^{s}$ given by $\|f\|_{s}=\left\|\Lambda^{s} f\right\|$ where $\Lambda^{2}=(-\Delta)$.

Our first goal is to prove the following Theorem which will be the consequence of the more general results given later in the text. In particular, it follows from Theorem 3.1 Theorem 6.1 and Corollary 8.2 when combined with Proposition 3.2 .

Theorem 1.1. Consider the forcing

$$
W(t, x)=W_{1}(t) \sin \left(x_{1}\right)+W_{2}(t) \cos \left(x_{1}\right)+W_{3}(t) \sin \left(x_{1}+x_{2}\right)+W_{4}(t) \cos \left(x_{1}+x_{2}\right),
$$

then for any $t>0$ and any finite dimensional subspace $S$ of $\mathbb{L}^{2}$, the law of the orthogonal projection $\Pi w(t, \cdot)$ of $w(t, \cdot)$ onto $S$ is absolutely continuous with respect 
to the Lebesgue measure on $S$. Furthermore, the density is $C^{\infty}$ and everywhere strictly positive.

A version of Theorem 1.1 for Galerkin approximations of (1.1) was one of the main ingredients of the ergodic and exponential mixing results proven in [EM01]. There the algebraic structure of the nonlinearity was exploited to show that the associated diffusion was hypoelliptic. Here we use similar observations on the algebraic structure generated by the vectorfields. However, new tools are required as there exists little theory applying Malliavin calculus in an infinite dimensional setting. Relevant exceptions are [HS81], [Oco88], and [EH01].

In [EH01], Malliavin calculus was used to establish the existence of a density when all but a finite number of degrees of freedom were forced. In contrast to the present paper, the technique developed there fundamentally required that only a finite number of directions are unforced. The ideas developed in the present paper could also likely be applied to the setting of [EH01].

An essential tool in our approach is a representation of the Malliavin covariance matrix through the solution of a backward (stochastic) partial differential equation, which was first invented by Ocone, see [Oco88], and which is particularly useful when dealing with certain classes of SPDEs, since in this case (as opposed to that of finite dimensional SDEs), the fundamental solution of the linearized equation cannot be easily inverted. Ocone used that representation in the case where the original equation is a so-called "bilinear SPDE" (that is both the coefficients of " $d t$ " and " $d W(t)$ " are linear in the solution). In contrast, we use it in the case of a nonlinear PDE with additive noise. It seems that these are the only two cases where Ocone's representation of the Malliavin matrix through a backward (S)PDE can be used, whithout being exposed to the trouble of handling a stochastic PDE involving anticipative stochastic integrals. In Ocone's case, the backward PDE is a stochastic one, while in our case it is a PDE with random coefficients.

There has been a lot of activity in recent years exploring the ergodic properties of the stochastic Navier-Stokes equations and other dissipative stochastic partial differential equations. The central new idea was to make use of the pathwise contractive properties of the dynamics on the small scales and the mixing/smoothing due to the stochastic forcing on the larger scales. In [Mat98] a determining modes type theorem (see [FP67] ) was developed in the stochastic setting. This showed how controlling the behavior of a finite number of low modes on a time interval of infinite length was sufficient to control the entire system. An important advance was made concurrently in [BKL01, EMS01, KS00], where it was shown that if all of the low modes were directly forced the system was ergodic. The first two covered the case of white in time forcing while the later considered impulsive forcing. The assumptions of these papers can be restated as : the diffusion is elliptic on the unstable subspace of the pathwise dynamics (see [Mat03] on this point of view). The present paper establishes the needed control on the low modes when a "partial 
hypoelliptic" assumption is satisfied. We show that the forcing need not excite directly all the unstable modes because the nonlinearity transmits the randomness to the non-directly excited unstable directions. Already, the results of this paper have been used in an essential way in [HM04] to prove the ergodicity of the stochastic Navier Stokes equations under mild, viscosity independent, assumptions on the geometry of the forcing.

This article is organized as follows: In section 2 we discuss the elements of Malliavin calculus needed in the paper. In particular, we give an alternative representation of the quadratic form associated to the Malliavin matrix. This representation is critical to the rest of the article. In section 3 we explore the structure of the nonlinearity as it relates to nondegeneracy of the Malliavin matrix which in turn implies the existence of a density. In section 4 , we prove an abstract lemma on the quadratic variation of non-adapted processes of a particular form which is the key to the results of the preceding section. In section [5, we discuss the relationship to brackets of vector fields and the usual proof of nondegeneracy of the Malliavin matrix. In doing so we sketch an alternative proof of the existence of a density. In section 6 we prove that the density, whose existence is given in section 3, is in fact $C^{\infty}$. This requires the abstract results of section 7 which amount to quantitative versions of the results in section 4 . Finally in Section 8 we prove that the density of the finite dimensional projections of $w(t)$ are everywhere positive under the same conditions which guarantee smoothness. We then give a number of concluding remarks and finish with five appendices containing technical estimates on the stochastic Navier Stokes equation. In particular, appendix C proves that the solution is smooth in the Malliavin sense and appendix E gives control of the Lipschitz constants in terms of various quantities associated to the solution.

\section{Representation of the Malliavin covariance matrix}

One way to solve the vorticity equation is by letting $w^{\prime}(t, x)=w(t, x)-W(t, x)$, and solving the resulting PDE with random coefficients for $w^{\prime}$. It easily follows from that approach that for each $t>0$, there exists a continuous map

$$
\Phi_{t}: C\left([0, t] ; \mathbb{R}^{\mathscr{Z}}\right) \rightarrow \mathbb{L}^{2},
$$

such that

$$
w(t)=\Phi_{t}\left(W_{[0, t]}\right) .
$$

In other words, the solution of equation 1.1 can be constructed pathwise. We shall exploit this in Section 8

For $k \in \mathscr{Z}_{*}, h \in L_{\text {loc }}^{2}\left(\mathbb{R}_{+}\right), t>0$, we define, if it exists, the Malliavin derivative of $w(t)$ in the direction $(k, h)$ as

$$
D^{k, h} w(t)=L^{2}\left(\Omega, \mathbb{L}^{2}\right)-\lim _{\varepsilon \longrightarrow 0} \frac{\Phi_{t}\left(W+\varepsilon H e_{k}\right)-\Phi_{t}(W)}{\varepsilon},
$$


where $H(t)=\int_{0}^{t} h(s) d s$. In fact, this convergence holds pathwise, and it is a Fréchet derivative.

We will show that the above derivative exists, for each $h \in L_{l o c}^{2}\left(\mathbb{R}_{+}\right)$, and moreover that for each $s \in[0, t]$ and $k \in \mathscr{Z}_{*}$ there exists a random element $V_{k, s}(t)$ in $\mathbb{L}^{2}$, such that

$$
D^{k, h} w(t)=\int_{0}^{t} V_{k, s}(t) h(s) d s .
$$

$V_{k, s}(t)$ is then identified with $D_{s}^{k} w(t) \stackrel{\text { def }}{=} D^{k, \delta_{s}} w(t)$ and solves equation 2.1 below.

Proposition 2.1. For each $s>0$ and $k \in \mathscr{Z}_{*}$, the linear parabolic PDE

$$
\left\{\begin{aligned}
\frac{\partial}{\partial t} V_{k, s}(t) & =v \Delta V_{k, s}(t)-B\left(w(t), V_{k, s}(t)\right)-B\left(V_{k, s}(t), w(t)\right), t \geq s \\
V_{k, s}(s) & =e_{k}
\end{aligned}\right.
$$

has a unique solution

$$
V_{k, s} \in C\left([s,+\infty) ; \mathbb{L}^{2}\right) \cap L_{l o c}^{2}\left([s,+\infty) ; \mathbb{H}^{1}\right) .
$$

Proof. See e.g. Constantin, Foias [CF88].

At times we will consider the linearized equation 2.1 with arbitrary initial conditions. We write $J_{s, t} \phi$ for the solution to 2.1) at time $t$ with initial condition $\phi$ at time $s$ less than $t$. In this notation $V_{k, s}(t)=J_{s, t} e_{k}$.

Furthermore, Lemma B.1 from the appendix implies that for all deterministic initial conditions $w(0), p \geq 1, \eta>0$, and $T<\infty$,

$$
\mathbb{E} \sup _{0 \leq s \leq t \leq T}\left\|V_{k, s}(t)\right\|^{2 p}<c \exp \left(\eta\|w(0)\|^{2}\right)
$$

for some $c=c(v, p, T, \eta)$.

Clearly, if $h \in L_{\text {loc }}^{2}\left(\mathbb{R}_{+}\right)$,

$$
V_{k, h}(t) \stackrel{\text { def }}{=} \int_{0}^{t} V_{k, s}(t) h(s) d s
$$

is the unique solution in $C\left([0,+\infty) ; \mathbb{L}^{2}\right) \cap L_{l o c}^{2}\left([0,+\infty) ; \mathbb{H}^{1}\right)$ of the parabolic PDE (2.2)

$$
\left\{\begin{aligned}
\frac{\partial V_{k, h}(t)}{\partial t} & =v \Delta V_{k, h}(t)-B\left(w(t), V_{k, h}(t)\right)-B\left(V_{k, h}(t), w(t)\right)+h(t) e_{k}, t \geq 0 \\
V_{k, h}(0) & =0
\end{aligned}\right.
$$

It is not hard to see that, in the sense of convergence in $L^{2}\left(\Omega ; \mathbb{L}^{2}\right)$,

$$
V_{k, h}(t)=\lim _{\varepsilon \rightarrow 0} \frac{\Phi_{t}\left(W+\varepsilon H e_{k}\right)-\Phi_{t}(W)}{\varepsilon} .
$$


It then follows that $w(t) \in H^{1}\left(\Omega, \mathbb{L}^{2}\right) \stackrel{\text { def }}{=}\left\{X: \Omega \rightarrow \mathbb{L}^{2}: \mathbb{E}\|X\|^{2}, \mathbb{E} \int_{0}^{t}\left\|D_{s}^{k} X\right\|^{2} d s<\right.$ $\infty$ for all $k \in \mathscr{Z}_{*}$ and finite $\left.t>0\right\}$ (see Nualart [Nua95] page 27 and 62 for more details). Furthermore, its associated infinite dimensional Malliavin covariance matrix is given by:

$$
\mathscr{M}(t)=\sum_{k \in \mathscr{Z}_{*}} \int_{0}^{t} V_{k, s}(t) \otimes V_{k, s}(t) d s
$$

that is to say it is the operator mapping $\phi \in \mathbb{L}^{2}$ to $\mathscr{M}(t)(\phi) \in \mathbb{L}^{2}$ given by

$$
\mathscr{M}(t)(\phi)=\sum_{k \in \mathscr{Z}_{*}} \int_{0}^{t}\left\langle V_{k, s}(t), \phi\right\rangle V_{k, s}(t) d s
$$

It follows from Theorem 2.1.2 in Nualart [Nua95] that Theorem 1.1 is a consequence of the fact that for each $\phi \in \mathbb{L}^{2}$ with $\phi \neq 0$,

$$
\langle\mathscr{M}(t) \phi, \phi\rangle=\sum_{k \in \mathscr{Z}_{*}} \int_{0}^{t}\left\langle V_{k, s}(t), \phi\right\rangle^{2} d s>0 \text { a.s. }
$$

We now want to give an alternative representation of this quantity, using a backward PDE which is the adjoint of equation (2.2).

Proposition 2.2. For each $t>0, \phi \in \mathbb{L}^{2}$, the linear backward parabolic PDE

$$
\left\{\begin{array}{r}
\frac{\partial}{\partial s} U^{t, \phi}(s)+v \Delta U^{t, \phi}(s)+B\left(w(s), U^{t, \phi}(s)\right)-C\left(U^{t, \phi}(s), w(s)\right)=0, \\
0 \leq s \leq t ; \\
U^{t, \phi}(t)=\phi,
\end{array}\right.
$$

has a unique solution

$$
U^{t, \phi} \in C\left([0, t] ; \mathbb{L}^{2}\right) \cap L^{2}\left([0, t) ; \mathbb{H}^{1}\right) .
$$

Here $C(\cdot, w(s))$ is the $\mathbb{L}^{2}$-adjoint of the time-dependent, linear operator $B(\cdot, w(s))$ and thus is defined by the relation $\langle B(u, w(s)), v\rangle=\langle C(v, w(s)), u\rangle$.

Proof. Same argument as in Proposition 2.1

As before Lemma B.1 from the appendix implies that there exist a positive constant $\eta$ so that for all deterministic initial conditions $w(0), \phi \in \mathbb{L}^{2}, p \geq 1$ and $T<\infty$

$$
\mathbb{E} \sup _{0 \leq s \leq t \leq T}\left\|U^{t, \phi}(s)\right\|^{2 p}<c\|\phi\|^{2 p} \exp \left(\eta\|w(0)\|^{2}\right) .
$$

for some $c=c(v, p, T, \eta)$.

Proposition 2.3. For each $k \in \mathscr{Z}_{*}$ and $\phi \in \mathbb{L}^{2}$, the function

$$
r \rightarrow\left\langle V_{k, s}(r), U^{t, \phi}(r)\right\rangle
$$

from $[s, t]$ into $\mathbb{R}$ is constant. 
Proof. We first show that this mapping belongs to $W^{1,1}(s, t ; \mathbb{R})$. It is clearly a continuous function and moreover

$$
V, U \in C\left([s, t] ; \mathbb{L}^{2}\right) \cap L^{2}\left((s, t) ; \mathbb{H}^{1}\right),
$$

hence by interpolation

$$
V, U \in L^{4}\left((s, t), \mathbb{H}^{\frac{1}{2}}\right) \cap L^{2}\left((s, t) ; \mathbb{H}^{1}\right) .
$$

Since we also know that

$$
w \in L^{4}\left((s, t) ; \mathbb{H}^{\frac{1}{2}}\right) \cap L^{2}\left((s, t) ; \mathbb{H}^{1}\right),
$$

we know that the products $\|U\|_{\frac{1}{2}}\|w\|_{1},\|V\|_{\frac{1}{2}}\|w\|_{1},\|w\|_{\frac{1}{2}}\|U\|_{1}$ and $\|w\|_{\frac{1}{2}}\|V\|_{1}$ all belong to $L^{\frac{4}{3}}((s, t) ; \mathbb{R})$. On the other hand, it follows from the fact that $\|\mathscr{K} U\|_{1}=$ $\|U\|$ and estimate (6.10) in Constantin, Foias [CF88] that

$$
\begin{array}{ll}
|\langle C(U, w), \psi\rangle| \leq c\|U\|_{\frac{1}{2}}\|w\|_{1}\|\psi\|_{\frac{1}{2}} & |\langle B(V, w), \psi\rangle| \leq c\|V\|_{\frac{1}{2}}\|w\|_{1} \mid\|\psi\|_{\frac{1}{2}} \\
|\langle B(w, U), \psi\rangle| \leq c\|w\|_{\frac{1}{2}}\|U\|_{1}\|\psi\|_{\frac{1}{2}} & |\langle B(w, V), \psi\rangle| \leq c\|w\|_{\frac{1}{2}}\|V\|_{1}\|\psi\|_{\frac{1}{2}} .
\end{array}
$$

Hence we conclude that $C(U, w), B(V, w), B(w, U)$ and $B(w, V)$ all belong to $L^{\frac{4}{3}}\left((s, t) ; \mathbb{H}^{-\frac{1}{2}}\right)$. From (2.1) and (2.5), we that that both $\frac{d}{d t} V$ and $\frac{d}{d t} U$ consist of three terms. The first belongs to $L^{2}\left((s, t) ; \mathbb{H}^{-1}\right)$ and the last two to $L^{\frac{4}{3}}\left((s, t) ; \mathbb{H}^{-\frac{1}{2}}\right)$. Hence $\left\langle V_{k, s}(r), \frac{d}{d r} U^{t, \phi}(r)\right\rangle$ and $\left\langle\frac{d}{d r} V_{k, s}(r), U^{t, \phi}(r)\right\rangle$ are in $L^{1}(s, t ; \mathbb{R})$ and the statement that

$$
r \rightarrow\left\langle V_{k, s}(r), U^{t, \phi}(r)\right\rangle
$$

is a.e. differentiable then follows a variant of Theorem 2, Chapter 18, section 1 in Dautray, Lions [DL88]. Moreover, for almost every $r$

$$
\begin{aligned}
\frac{d}{d r}\langle V(r), U(r)\rangle & =\langle A(w(r)) V(r), U(r)\rangle-\left\langle V(r), A^{*}(w(r)) U(r)\right\rangle \\
& =0
\end{aligned}
$$

where $A(w(t))$ is the linear operator on the right handside of 2.1 and $A^{*}(w(t))$ is its $\mathbb{L}^{2}$-adjoint. The result follows.

We can now rewrite the Malliavin covariance matrix using $U$ in place of $V$. For a fixed $\phi$, this is an improvement as $U^{t, \phi}(r)$ is a single solution to a PDE while $V_{k, t}(r)$ is a continuum of solutions indexed by the parameter $s$.

Corollary 2.4. For any $\phi \in \mathbb{L}^{2}$,

$$
\langle\mathscr{M}(t) \phi, \phi\rangle=\sum_{k \in \mathscr{Z}_{*}} \int_{0}^{t}\left\langle e_{k}, U^{t, \phi}(s)\right\rangle^{2} d s .
$$

Proof. This follows from the fact that for any $0 \leq s \leq t$ and $k \in \mathscr{Z}_{*}$,

$$
\left\langle V_{k, s}(s), U^{t, \phi}(s)\right\rangle=\left\langle V_{k, s}(t), U^{t, \phi}(t)\right\rangle
$$

i.e.

$$
\left\langle e_{k}, U^{t, \phi}(s)\right\rangle=\left\langle V_{k, s}(t), \phi\right\rangle
$$


From this corollary, one immediately deduces the following result.

Corollary 2.5. Assume that for some fixed $\phi \in \mathbb{L}^{2}$,

$$
\langle\mathscr{M}(t) \phi, \phi\rangle=0
$$

on a subset $\Omega_{1}$ of $\Omega$. Then for all $k \in \mathscr{Z}_{*}$ and $s \leq t,\left\langle e_{k}, U^{t, \phi}(s)\right\rangle=0$ on $\Omega_{1}$. In particular, $\left\langle e_{k}, \phi\right\rangle=0$.

\section{Hypoellipticity}

\subsection{Final Assumptions and Main Existence Result}

We define $\mathscr{Z}_{0}$ to be the symmetric part of the forcing set $\mathscr{Z}_{*}$ given by $\mathscr{Z}_{0}=$ $\mathscr{Z}_{*} \cap\left(-\mathscr{Z}_{*}\right)$ and then the collection

$$
\mathscr{Z}_{n}=\left\{\ell+j \in \mathbb{Z}_{0}^{2}: j \in \mathscr{Z}_{0}, \quad \ell \in \mathscr{Z}_{n-1} \text { with } \ell^{\perp} \cdot j \neq 0,\|j\| \neq\|\ell\|\right\}
$$

and lastly,

$$
\mathscr{Z}_{\infty}=\bigcup_{n=1}^{\infty} \mathscr{Z}_{n} .
$$

Notice that the above union starts at one and that the $\mathscr{Z}_{n}$ are symmetric in that $\mathscr{Z}_{n}=-\mathscr{Z}_{n}$. This follows by recurrence, starting with $\mathscr{Z}_{0}=-\mathscr{Z}_{0}$. We are mainly concerned with the case where $\mathscr{Z}_{0}=\mathscr{Z}_{*}$ as this corresponds to noise which is stationary in $x$. We now can state the main theorem. Defining

$$
S_{0}=\operatorname{Span}\left(e_{k}: k \in \mathscr{Z}_{*}\right) ; S_{n}=\operatorname{Span}\left(e_{k}: k \in \bigcup_{j=1}^{n} \mathscr{Z}_{j} \cup \mathscr{Z}_{*}\right), n \in\{1,2, \ldots, \infty\},
$$

we have the following result which implies the first part of Theorem 1.1 in the case when $S_{\infty}=\mathbb{L}^{2}$.

Theorem 3.1. For any $t>0$ and any finite dimensional subspace $S$ of $S_{\infty}$, the law of the orthogonal projection $\Pi w(t, \cdot)$ of $w(t, \cdot)$ onto $S$ is absolutely continuous with respect to the Lebesgue measure on $S$.

The above result guarantees an absolutely continuous density on finite dimensional subsets of $S_{\infty}$. However, it does not imply the lack of density for other subsets as in constructing $S_{\infty}$ we have only used part of the available information. In the proof below, it will become clear that we make use only of the directions generated by frequencies where both the sin and cos are stochastically forced. We do this in the name of simplicity and utility. Verifying any more complicated condition was difficult. However, as translation invariance implies that both the sin and cos mode of a given frequency are forced, it seems a reasonable compromise. In the end, we are primarily interested in producing conditions which give insight as to how the nonlinearity spreads the randomness. In particular we now give an 
easy Proposition which in conjunction with Theorem 3.1 proves the first part of Theorem 1.1 given in the introduction. After that we will quote a more general result from [HM04] which is proven using similar ideas to those below.

Proposition 3.2. If $\{(0,1),(1,1)\} \subset \mathscr{Z}_{0}$ then $S_{\infty}=\mathbb{L}^{2}$.

Proof. Clearly by adding and subtracting the vectors $(0,1)$ and $(1,1)$, one can generate all of $\mathbb{Z}_{0}^{2}$. The only question is whether the conditions $\ell^{\perp} \cdot j \neq 0$ and $\|j\| \neq\|\ell\|$ from the definition of $\mathscr{Z}_{n}$ ever create a situation which blocks continuing generating the lattice. First notice that $(1,0)=(1,1)-(0,1),(-1,0)=(0,1)-(1,1)$ and $(-1,1)=(-1,0)-(1,1)+(0,1)+(1,1)$ and if these moves are made from left to right none of the restrictions are violated. Hence all of the vectors of the same length as the vectors in $\mathscr{Z}_{0}$ can be reached and henceforth the restriction $\|j\| \neq\|\ell\|$ will not be binding. The requirement that $\ell^{\perp} \cdot j \neq 0$ does not cause a problem. The line $\ell^{\perp} \cdot(0,1)=0$ can be approached from above and does not obstruct generating the rest of the lattice. The line $\ell^{\perp} \cdot(1,1)=0$ does separate the lattice. However we know we can reach $(1,0)$ and have the point $(0,1)$ to start with. Hence we can reach all of the points on either side of the line. Those on the line can be reached from points on either side.

It is clear from the preceding lemma that many other choices of forcing will also lead to $S_{\infty}=\mathbb{L}^{2}$. For instance if $\{(1,0),(1,1)\} \subset \mathscr{Z}_{0}$ then $S_{\infty}=\mathbb{L}^{2}$. It is also interesting to force a collection of modes distant from the origin and allow the noise to propagate both up to the large scales and down to still smaller scales. We now give a simple proposition giving sufficient conditions in such a setting.

Proposition 3.3. Let $M, K \in \mathbb{N}$ with $M, K>2$ and $\|M-K\|>2$. Then if $\{(M+$ $1,0),(M, 0),(0, K+1),(0, K)\} \subset \mathscr{Z}_{0}, S_{\infty}=\mathbb{L}^{2}$.

Proof. The idea is to use $(M+1,0)-(M, 0)=(1,0),(M, 0)-(M+1,0)=(-1,0)$, $(0, K+1)-(0, K)=(0,1)$ and $(0, K)-(0, K+1)=(0,-1)$ in order to generate the whole lattice. The only difficulty could be the above restrictions. The restrictions of the form $\ell^{\perp} \cdot j \neq 0$ only prevent applying $(M+1,0)-(M, 0)$ or $(M, 0)-(M+1,0)$ to points on the $x$-axis and $(0, K)-(0, K+1)$ and $(0, K+1)-(0, K)$ to points on the $y$-axis. However this is not a serious restriction as all of the points on the $x$-axis can be reached by moving down from above and all of the points on the $y$-axis can be reached by moving horizontally. Furthermore the $y$-axis can be crossed by using strictly horizontal moves. The only remaining restriction is the points $k \in \mathbb{Z}_{0}^{2}$ with $\|k\| \in\{K, K+1, M, M+1\}$. For example assume that $\|k\|=K$ and one wanted to move to the left by applying $(0, K)-(0, K+1)$. While this direct move is illegal, one can accomplish the same effect by moving up then left and finally down. The requirement that $\|M-K\|>2$ ensures that we will not be blocked from moving up using $(M+1,0)-(M, 0)$ given that $k=\|K\|$. Once we have moved up, we will be free to move to the left and then back down. The other cases are analogous.

Guided by these results, in [HM04] the following is proven: 
Proposition 3.4. One has $S_{\infty}=\mathbb{L}^{2}$ if and only if:

(1) Integer linear combinations of elements of $\mathscr{Z}_{0}$ generate $\mathbb{Z}_{0}^{2}$.

(2) There exist at least two elements in $\mathscr{Z}_{0}$ with unequal euclidean norm.

This gives a very satisfactory characterization of the setting when $S_{\infty}=\mathbb{L}^{2}$ which is the case of primary interest.

\subsection{Proof of Theorem 3.1}

Since we already know that $w(t) \in H^{1}\left(\Omega, \mathbb{L}^{2}\right)$, Theorem 3.1 follows from Theorem 2.1.2 in Nualart [Nua95] and the fact that for any $\phi \in S_{\infty}, \phi \neq 0$,

$$
\langle\mathscr{M}(t) \phi, \phi\rangle>0 \text { a.s. }
$$

Hence to prove Theorem 3.1 it suffices to show that

Proposition 3.5. There exists a subset $\Omega_{1} \subset \Omega$ of full measure so that on $\Omega_{1}$ if $\langle\mathscr{M}(t) \phi, \phi\rangle=0$ for some $\phi \in \mathbb{L}^{2}$ then $\Pi_{\infty} \phi=0$ where $\Pi_{\infty}$ is the $\mathbb{L}^{2}$-orthogonal projection onto $S_{\infty}$.

Notice that Proposition 3.5 is equivalent to

$$
\mathbb{P}\left(\bigcap_{\substack{\phi \in \mathbb{L}^{2} \\ \Pi_{\infty} \phi \neq 0}}\{\langle\mathscr{M}(t) \phi, \phi\rangle>0\}\right)=1 .
$$

To prove the proposition we need to better understand the structure of the equations. To this end, we now write the equations for the spatial Fourier coefficients of $U$ and $w$ to better expose the interactions between the systems various degrees of freedom. In this and the general structures of the nonlinearity exploited, we follow E, Mattingly [EM01]; however, the tact of the analysis is different. (We also take the chance to correct a small error in [EM01]. There the summation was restricted to modes in the first quadrant, when it should have ranged over the entire upper-half plane.) Again setting $w(t, x)=\sum_{k \in \mathbb{Z}_{0}^{2}} \alpha_{k}(t) e_{k}$, we have for $\ell \in \mathbb{Z}_{0}^{2}$

$$
\begin{aligned}
\frac{d}{d t} \alpha_{\ell}(t)+v\|\ell\|^{2} \alpha_{\ell}(t)+ & \frac{1}{2} \sum_{(j, k, \ell) \in \mathscr{I}_{+}} c(j, k) \alpha_{j}(t) \alpha_{k}(t) \\
& -\frac{1}{2} \sum_{(j, k, \ell) \in \mathscr{I}_{-}} c(j, k) \alpha_{j}(t) \alpha_{k}(t)=\mathbf{1}_{\mathscr{Z}_{*}}(\ell) \frac{d}{d t} W_{\ell}(t)
\end{aligned}
$$

where $\mathbf{1}_{\mathscr{Z}_{*}}$ is the indicator function of $\mathscr{Z}_{*}, c(j, k)=\frac{1}{2}\left(j^{\perp} \cdot k\right)\left(\|j\|^{-2}-\|k\|^{-2}\right)$ and

$$
\begin{aligned}
\mathscr{I}_{+}= & \left\{(j, k, \ell) \in\left(\mathbb{Z}_{+}^{2}, \mathbb{Z}_{-}^{2}, \mathbb{Z}_{+}^{2}\right) \cup\left(\mathbb{Z}_{-}^{2}, \mathbb{Z}_{+}^{2}, \mathbb{Z}_{+}^{2}\right) \cup\left(\mathbb{Z}_{+}^{2}, \mathbb{Z}_{+}^{2}, \mathbb{Z}_{-}^{2}\right) \mid k+j+\ell=0\right\} \\
& \cup\left\{(j, k, \ell) \in\left(\mathbb{Z}_{+}^{2}, \mathbb{Z}_{-}^{2}, \mathbb{Z}_{+}^{2}\right) \cup\left(\mathbb{Z}_{-}^{2}, \mathbb{Z}_{-}^{2}, \mathbb{Z}_{-}^{2}\right) \cup\left(\mathbb{Z}_{+}^{2}, \mathbb{Z}_{+}^{2}, \mathbb{Z}_{-}^{2}\right) \mid \ell=j-k\right\} \\
& \cup\left\{(j, k, \ell) \in\left(\mathbb{Z}_{-}^{2}, \mathbb{Z}_{+}^{2}, \mathbb{Z}_{+}^{2}\right) \cup\left(\mathbb{Z}_{-}^{2}, \mathbb{Z}_{-}^{2}, \mathbb{Z}_{-}^{2}\right) \cup\left(\mathbb{Z}_{+}^{2}, \mathbb{Z}_{+}^{2}, \mathbb{Z}_{-}^{2}\right) \mid \ell=k-j\right\} \\
\mathscr{I}_{-}= & \left\{(j, k, \ell) \in\left(\mathbb{Z}_{-}^{2}, \mathbb{Z}_{+}^{2}, \mathbb{Z}_{+}^{2}\right) \cup\left(\mathbb{Z}_{+}^{2}, \mathbb{Z}_{-}^{2}, \mathbb{Z}_{+}^{2}\right) \cup\left(\mathbb{Z}_{-}^{2}, \mathbb{Z}_{-}^{2}, \mathbb{Z}_{-}^{2}\right) \mid \ell=j+k\right\}
\end{aligned}
$$


Setting

$$
U^{t, \phi}(s, x)=\sum_{k \in \mathbb{Z}_{0}^{2}} \beta_{k}^{\phi}(s) e_{k}(x), \text { and } \phi(x)=\sum_{k \in \mathbb{Z}_{0}^{2}} \phi_{k} e_{k}(x),
$$

we also have the backward equations

$$
\begin{aligned}
\frac{d}{d s} \beta_{\ell}^{\phi}(s) & =v\|\ell\|^{2} \beta_{\ell}^{\phi}(s)+\sum_{(j, k, \ell) \in \mathscr{I}_{+}^{*}} c(j, \ell) \alpha_{j}(t) \beta_{k}^{\phi}(t) \\
& -\sum_{(j, k, \ell) \in \mathscr{I}_{-}^{*}} c(j, \ell) \alpha_{j}(t) \beta_{k}^{\phi}(t) \quad s<t, \\
\beta_{\ell}^{\phi}(t) & =\phi_{\ell} .
\end{aligned}
$$

where

$$
\begin{aligned}
\mathscr{I}_{+}^{*}= & \left\{(j, k, \ell) \in\left(\mathbb{Z}_{+}^{2}, \mathbb{Z}_{+}^{2}, \mathbb{Z}_{-}^{2}\right) \cup\left(\mathbb{Z}_{-}^{2}, \mathbb{Z}_{+}^{2}, \mathbb{Z}_{+}^{2}\right) \cup\left(\mathbb{Z}_{+}^{2}, \mathbb{Z}_{-}^{2}, \mathbb{Z}_{+}^{2}\right) \mid j+k+\ell=0\right\} \\
& \cup\left\{(j, k, \ell) \in\left(\mathbb{Z}_{+}^{2}, \mathbb{Z}_{+}^{2}, \mathbb{Z}_{-}^{2}\right) \cup\left(\mathbb{Z}_{-}^{2}, \mathbb{Z}_{-}^{2}, \mathbb{Z}_{-}^{2}\right) \cup\left(\mathbb{Z}_{+}^{2}, \mathbb{Z}_{-}^{2}, \mathbb{Z}_{+}^{2}\right) \mid \ell=j-k\right\} \\
& \cup\left\{(j, k, \ell) \in\left(\mathbb{Z}_{-}^{2}, \mathbb{Z}_{+}^{2}, \mathbb{Z}_{+}^{2}\right) \cup\left(\mathbb{Z}_{-}^{2}, \mathbb{Z}_{-}^{2}, \mathbb{Z}_{-}^{2}\right) \cup\left(\mathbb{Z}_{+}^{2}, \mathbb{Z}_{-}^{2}, \mathbb{Z}_{+}^{2}\right) \mid \ell=j+k\right\} \\
\mathscr{I}_{-}^{*}= & \left\{(j, k, \ell) \in\left(\mathbb{Z}_{-}^{2}, \mathbb{Z}_{+}^{2}, \mathbb{Z}_{+}^{2}\right) \cup\left(\mathbb{Z}_{+}^{2}, \mathbb{Z}_{+}^{2}, \mathbb{Z}_{-}^{2}\right) \cup\left(\mathbb{Z}_{-}^{2}, \mathbb{Z}_{-}^{2}, \mathbb{Z}_{-}^{2}\right) \mid \ell=k-j\right\} .
\end{aligned}
$$

We now continue the proof of Proposition 3.5. Notice that the $\beta_{\ell}^{\phi}$ are continuous in time for every $\ell \in \mathscr{Z}_{0}$, every $\phi \in \mathbb{L}^{2}$ and every realization of the stochastic forcing. Hence if $\beta_{\ell}^{\phi} \equiv 0$ for some realization of noise, then $\phi_{\ell}=0$. (The notation $x \equiv 0$ means $x(s)=0, s \in[0, t)$.) Thus to prove the lemma it would be sufficient to show that there existed a fixed set $\Omega_{1}$ with positive probability so that for any $\phi \in S_{\infty}$, if $\langle\mathscr{M}(t) \phi, \phi\rangle=0$ on $\Omega_{1}$ then $\beta_{\ell}^{\phi} \equiv 0$ for all $\ell \in \mathscr{Z}_{*} \cup \mathscr{Z}_{\infty}$. This will be proven inductively.

The base case of the induction is given by Corollary 2.5 In the present notation, it simply says that for any $\omega \in \Omega$ if $\langle\mathscr{M}(t) \phi, \phi\rangle=0$ for some $\phi \in \mathbb{L}^{2}$ then $\beta_{\ell}^{\phi} \equiv 0$ for all $\ell \in \mathscr{Z}_{*}$. In particular, for any $\omega \in \Omega$ if $\langle\mathscr{M}(t) \phi, \phi\rangle=0$ then $\beta_{\ell}^{\phi} \equiv \beta_{-\ell}^{\phi} \equiv 0$ for all $\ell \in \mathscr{Z}_{0}$. The proof of Theorem 3.1 would then be complete if we show that there exists a single subset $\Omega_{1} \subset \Omega$ of full measure so that if $\beta_{\ell}^{\phi} \equiv \beta_{-\ell}^{\phi} \equiv 0$ on $\Omega_{1}$ for all $\ell \in \mathscr{Z}_{n}$ then $\beta_{\ell}^{\phi} \equiv \beta_{-\ell}^{\phi} \equiv 0$ on $\Omega_{1}$ for all $\ell \in \mathscr{Z}_{n+1}$. This inductive step is given by the next lemma, which once proved completes the proof of Theorem 3.1

Lemma 3.6. There exists a fixed subset $\Omega_{1}$ of full measure so that for any $\phi \in \mathbb{L}^{2}$ and $\ell \in \mathbb{Z}_{0}^{2}$ if $\beta_{\ell}^{\phi} \equiv 0$ and $\beta_{-\ell}^{\phi} \equiv 0$ on $\Omega_{1}$ then for all $j \in \mathscr{Z}_{0}$ such that $j^{\perp} \cdot \ell \neq 0$ and $\|j\| \neq \| \ell$

$$
\beta_{\ell+j}^{\phi} \equiv \beta_{-(\ell+j)}^{\phi} \equiv \beta_{\ell-j}^{\phi} \equiv \beta_{j-\ell}^{\phi} \equiv 0
$$

on $\Omega_{1}$. 
Proof. We begin with some simple observations which will be critical shortly. Notice that from 3.3 - 3.4) one sees that for $\ell \in \mathscr{Z}_{*}, \alpha_{\ell}(s)$ has the form

$$
\alpha_{\ell}(s)=\alpha_{\ell}(0)+\int_{0}^{s} \gamma_{\ell}(r) d r+W_{\ell}(s)
$$

where the $\gamma_{\ell}$ are some stochastic processes depending on the initial conditions and noise realizations. Hence these coordinates are the sum of a Brownian Motion and a part which has finite first variation and is continuous in time for all $\omega \in \Omega$.

Similarly, for $\ell \notin \mathscr{Z}_{*}$,

$$
\alpha_{\ell}(s)=\alpha_{\ell}(0)+\int_{0}^{s} \gamma_{\ell}(r) d r
$$

and hence these coordinates are continuous and have finite first variation in time for every $\omega \in \Omega$ as they are not directly forced. Similarly notice that $\beta_{\ell}^{\phi}$ is continuous and of finite first variation. In particular, we emphasis that these properties of $\alpha_{\ell}$ and $\beta_{\ell}^{\phi}$ hold on all of $\Omega$ for all $\ell \in \mathbb{Z}_{0}^{2}$ and $\phi \in \mathbb{L}^{2}$.

Now, if $\beta_{\ell}^{\phi} \equiv 0$ or $\beta_{-\ell}^{\phi} \equiv 0$ then $\frac{d}{d s} \beta_{\ell}^{\phi}(s) \equiv 0$ or $\frac{d}{d s} \beta_{-\ell}^{\phi}(s) \equiv 0$ respectively as the coordinates are constant. Notice that from 3.4 - 3.6 these derivatives have the form

$$
X(s)+\sum_{k \in \mathscr{Z}_{*}} Y_{k}(s) W_{k}(s)
$$

where the $X$ and $Y_{k}$ are continuous and bounded variation processes. Also notice that they are not adapted to the past of the $W_{k}$ 's ! Nonetheless, it follows from Lemma 4.1 in the next section that if $\left\{X(\cdot), Y_{k}(\cdot): k \in \mathscr{Z}_{*}\right\}$ are continuous and of bounded variation, then

$$
X(s)+\sum_{k \in \mathscr{Z}_{*}} Y_{k}(s) W_{k}(s)=0,0 \leq s \leq t,
$$

implies that

$$
Y_{k}(s)=0, \text { for all } 0 \leq s \leq t \text { and } k \in \mathscr{Z}_{*}
$$

on a set $\Omega_{1} \subset \Omega$, of full measure, which does not depend on $\ell, k$ or $\phi$, and hence we can use a single exceptional set for all of the steps in the induction. To summarize, we have shown that there is a single fixed set $\Omega_{1} \subset \Omega$, of full measure, so that for any $\phi \in \mathbb{L}^{2}$ if $\beta_{\ell}^{\phi} \equiv \beta_{-\ell}^{\phi} \equiv 0$ on $\Omega_{1}$ then $Y_{k} \equiv 0$ for all $k \in \mathscr{Z}_{*}$. We now identify the $Y_{k}$ to discover what (3.8) implies.

Define $|\ell|= \pm \ell$ depending on whether $\ell \in \mathbb{Z}_{ \pm}^{2}$ and $\operatorname{sgn}(\ell)= \pm 1$ depending on whether $\ell \in \mathbb{Z}_{ \pm}^{2}$. (Care should be taken not to confuse $\|\ell\|$ which is in $\mathbb{R}_{+}$with $|\ell|$ 
which is in $\mathbb{Z}_{+}^{2}$.) Then from (3.3) - (3.4), we see that for each $\ell \in \mathbb{Z}_{0}^{2}$

$$
\begin{aligned}
\frac{d}{d s} \beta_{\ell}^{\phi}(s)=X_{\ell}(s)+ & \sum_{\substack{j \in \mathscr{Z}_{*} \\
(j, \ell) \in I_{S}}} c(j, \ell)\left[\beta_{-|\ell-j|}^{\phi}(s)+\operatorname{sgn}(\ell) \beta_{-|\ell+j|}^{\phi}(s)\right] W_{j}(s) \\
+ & \sum_{\substack{j \in \mathscr{Z}_{*} \\
(j, \ell) \in I_{A}}} c(j, \ell)\left[\beta_{|\ell-j|}^{\phi}(s)-\operatorname{sgn}(\ell+j) \beta_{|\ell+j|}^{\phi}(s)\right] W_{j}(s),
\end{aligned}
$$

where $I_{A}=\left(\mathbb{Z}_{+}^{2}, \mathbb{Z}_{-}^{2}\right) \cup\left(\mathbb{Z}_{-}^{2}, \mathbb{Z}_{+}^{2}\right), I_{S}=\left(\mathbb{Z}_{+}^{2}, \mathbb{Z}_{+}^{2}\right) \cup\left(\mathbb{Z}_{-}^{2}, \mathbb{Z}_{-}^{2}\right)$, and $X_{\ell}(s)$ is a continuous stochastic process with bounded variation. Hence by Lemma 4.1 we obtain that terms in brackets in the above equation are identically zero.

Recall that by assumption $\frac{d}{d s} \beta_{\ell}^{\phi}(s)=0, \frac{d}{d s} \beta_{-\ell}^{\phi}(s)=0$ and $\{j,-j\} \subset \mathscr{Z}_{*}$. Without loss of generality, we assume that $\ell, j \in \mathbb{Z}_{+}$since this can always be achieved be renaming $\ell$ and $j$. The preceding reasoning using Lemma 4.1 applied to $(j, \ell)$, $(-j,-\ell),(-j, \ell)$, and $(j,-\ell)$ implies respectively that

$$
\begin{aligned}
c(j, \ell)\left[\beta_{-|\ell-j|}^{\phi}(s)+\beta_{-|\ell+j|}^{\phi}(s)\right] & =0 \\
c(j, \ell)\left[\beta_{-|\ell-j|}^{\phi}(s)-\beta_{-|\ell+j|}^{\phi}(s)\right] & =0 \\
c(j, \ell)\left[\operatorname{sgn}(\ell-j) \beta_{|\ell-j|}^{\phi}(s)-\beta_{|\ell+j|}^{\phi}(s)\right] & =0 \\
c(j, \ell)\left[\operatorname{sgn}(\ell-j) \beta_{|\ell-j|}^{\phi}(s)+\beta_{|\ell+j|}^{\phi}(s)\right] & =0
\end{aligned}
$$

for all $s<t$ on a subset of $\Omega_{1}$ of full measure. Provided that $j^{\perp} \cdot \ell \neq 0$ and $\|j\| \neq\|\ell\|$, one has that $c(\ell, j) \neq 0$. Hence the left-hand-sides are linearly independent and one concludes that $\beta_{\ell-j}^{\phi} \equiv \beta_{\ell+j}^{\phi} \equiv \beta_{j-\ell}^{\phi} \equiv \beta_{-(\ell+j)}^{\phi} \equiv 0$ on a subset of $\Omega_{1}$ of full measure.

We now collect some of the information from the preceding proof for later use.

Proposition 3.7. Let $U^{\phi, t}$ be the solution of (2.5) for any choice of terminal condition $\phi$ and terminal time $t$. Recall the definition of $S_{n}$ from (3.1). Let $\Pi_{0}$ be the projection onto $S_{0}$ and $\Pi_{0}^{\perp}$ its orthogonal complement. Then for $s<t$

$$
\frac{\partial}{\partial s} U^{\phi, t}(s)=X^{\phi}(s)+\sum_{j \in \mathscr{Z}_{*}} Y_{j}^{\phi}(s) W_{j}(s)
$$

where

$$
\begin{aligned}
& X^{\phi}(s)=-v \Delta U^{\phi, t}(s)-B\left(\Pi_{0}^{\perp} w(s), U^{\phi, t}(s)\right)+C\left(U^{\phi, t}(s), \Pi_{0}^{\perp} w(s)\right) \\
&-B\left(R(s), U^{\phi, t}(s)\right)+C\left(U^{\phi, t}(s), R(s)\right) \\
& R(s)= \Pi_{0} w(0)+\int_{0}^{s} v \Delta \Pi_{0} w(r)+\Pi_{0} B(w(r), w(r)) d r \\
& Y_{j}^{\phi}(s)=-B\left(e_{j}, U^{\phi, t}(s)\right)+C\left(U^{\phi, t}(s), e_{j}\right)
\end{aligned}
$$


For all $\ell, j \in \mathbb{Z}_{+}^{2}$, we have

$$
\begin{aligned}
\left\langle Y_{j}^{\phi}(s), e_{\ell}\right\rangle & =\pi^{2} c(j, \ell)\left[\beta_{-|\ell-j|}^{\phi}(s)+\beta_{-(\ell+j)}^{\phi}(s)\right] \\
\left\langle Y_{-j}^{\phi}(s), e_{-\ell}\right\rangle & =\pi^{2} c(j, \ell)\left[\beta_{-|\ell-j|}^{\phi}(s)-\beta_{-(\ell+j)}^{\phi}(s)\right] \\
\left\langle Y_{-j}^{\phi}(s), e_{\ell}\right\rangle & =\pi^{2} c(j, \ell)\left[\operatorname{sgn}(\ell-j) \beta_{|\ell-j|}^{\phi}(s)-\beta_{\ell+j}^{\phi}(s)\right] \\
\left\langle Y_{j}^{\phi}(s), e_{-\ell}\right\rangle & =-\pi^{2} c(j, \ell)\left[\operatorname{sgn}(\ell-j) \beta_{|\ell-j|}^{\phi}(s)+\beta_{\ell+j}^{\phi}(s)\right] .
\end{aligned}
$$

\section{A Quadratic Variation Lemma}

The following lemma is the main technical result used to prove the existence of a density.

Lemma 4.1. Let $\mathscr{A}$ be a collection of real valued stochastic processes such that there exists a fixed subset $\Omega_{\mathscr{A}} \subset \Omega$ of full measure such that on $\Omega_{\mathscr{A}}$ any element of $\mathscr{A}$ is continuous and has finite first variation.

Fix a finite collection $\left\{W_{1}, \ldots, W_{N}\right\}$ of independent Wiener processes and a sequence of partitions $\left\{s_{j}^{n}\right\}_{j=0}^{m(n)}$, with $s_{j+1}^{n}-s_{j}^{n} \rightarrow 0$ as $n \rightarrow \infty$ and

$$
0=s_{1}^{n} \leq \cdots \leq s_{m(n)}^{n}=t .
$$

Then there exists a fix subset $\Omega^{\prime} \subset \Omega_{\mathscr{A}}$ of full measure and a fixed subsequence of partitions $\left\{t_{j}^{n}\right\}_{j=0}^{k(n)}$ of $\left\{s_{j}^{n}\right\}_{j=0}^{m(n)}$ so that if

$$
Z(s)=X(s)+\sum_{i=1}^{N} Y_{i}(s) W_{i}(s)
$$

with $X, Y_{1}, \ldots, Y_{N} \in \mathscr{A}$ then on the set $\Omega^{\prime}$

$$
\sum_{j=1}^{k(n)}\left|Z\left(t_{j}^{n}\right)-Z\left(t_{j-1}^{n}\right)\right|^{2} \longrightarrow \sum_{i=1}^{N} \int_{0}^{t} Y_{i}^{2}(s) d \text { s as } n \rightarrow \infty .
$$

To prove this lemma we will invoke the following auxiliary results whose proofs will be given after the proof of Lemma 4.1

Lemma 4.2. Let $\{W(s), 0 \leq s \leq t\}$ be a standard Brownian motion and $\left\{s_{j}^{n}\right\}_{j=0}^{m(n)} a$ sequence of partitions as in Lemma 4.1] The sequence of measures

$$
\left\{\sum_{j=1}^{m(n)}\left(W\left(s_{j}^{n}\right)-W\left(s_{j-1}^{n}\right)\right)^{2} \delta_{s_{j-1}^{n}}, n=1,2 \ldots\right\}
$$

converges weakly as $n \rightarrow \infty$ to the Lebesgue measure on $[0, t]$, in probability. 
Lemma 4.3. Let $\{W(s), 0 \leq s \leq t\}$ and $\{\bar{W}(s), 0 \leq s \leq t\}$ be two mutually independent Brownian motions and $\left\{s_{j}^{n}\right\}_{j=0}^{m(n)}$ a sequence of partitions as in Lemma 4.1] The sequence of signed measures

$$
\left\{\sum_{j=1}^{m(n)}\left(W\left(s_{j}^{n}\right)-W\left(s_{j-1}^{n}\right)\right)\left(\bar{W}\left(s_{j}^{n}\right)-\bar{W}\left(s_{j-1}^{n}\right)\right) \delta_{s_{j-1}^{n}}, n=1,2, \ldots\right\}
$$

converges weakly to zero in probability as $n \rightarrow \infty$.

Proof of Lemma 4.1] In light of Lemma 4.2 and 4.3, since the collection of Brownian Motions is finite we can select a single set of full measure $\Omega^{\prime} \subset \Omega_{\mathscr{A}}$ and a single subsequence of partitions $\left\{t_{j}^{n}\right\}_{j=0}^{k(n)}$ such that the weak convergences given in Lemma 4.2 and 4.3 hold on $\Omega^{\prime}$. Furthermore we can assume that on $\Omega^{\prime}$ the $W_{i}(s)$ are continuous and have quadratic variation $s$. For notational brevity, we will write $t_{j}$ instead of $t_{j}^{n}$.

Consider the quantity $\sum_{j}\left|Z\left(t_{j}\right)-Z\left(t_{j-1}\right)\right|^{2}$. If we express it in terms of the $X, Y$ and $W$ 's we note that it contains four types of terms

$$
\begin{aligned}
& \sum_{j}\left|X\left(t_{j}\right)-X\left(t_{j-1}\right)\right|^{2} \\
& \sum_{j}\left|Y\left(t_{j}\right) W\left(t_{j}\right)-Y\left(t_{j-1}\right) W\left(t_{j-1}\right)\right|^{2} \\
& \sum_{j}\left(X\left(t_{j}\right)-X\left(t_{j-1}\right)\right)\left(Y\left(t_{j}\right) W\left(t_{j}\right)-Y\left(t_{j-1}\right) W\left(t_{j-1}\right)\right) \\
& \sum_{j}\left(Y\left(t_{j}\right) W\left(t_{j}\right)-Y\left(t_{j-1}\right) W\left(t_{j-1}\right)\right)\left(\bar{Y}\left(t_{j}\right) \bar{W}\left(t_{j}\right)-\bar{Y}\left(t_{j-1}\right) \bar{W}\left(t_{j-1}\right)\right)
\end{aligned}
$$

where $W$ and $\bar{W}$ are mutually independent scalar Brownian motions. The first and third terms are easily shown to tend to zero on $\Omega^{\prime}$ as $n \rightarrow \infty$, since $X$ is of bounded variation, and $X, Y$ and $W$ are continuous.

Consider the second term :

$$
\begin{aligned}
& \sum_{j}\left|Y\left(t_{j}\right) W\left(t_{j}\right)-Y\left(t_{j-1}\right) W\left(t_{j-1}\right)\right|^{2}=\sum_{j} Y\left(t_{j-1}\right)^{2}\left(W\left(t_{j}\right)-W\left(t_{j-1}\right)\right)^{2} \\
& +\sum_{j} W^{2}\left(t_{j}\right)\left(Y\left(t_{j}\right)-Y\left(t_{j-1}\right)\right)^{2} \\
& +2 \sum_{j} W\left(t_{j}\right) Y\left(t_{j-1}\right)\left(W\left(t_{j}\right)-W\left(t_{j-1}\right)\right) \times\left(Y\left(t_{j}\right)-Y\left(t_{j-1}\right)\right)
\end{aligned}
$$

Again on $\Omega^{\prime}$, the second and last terms above tend to zero, and

$$
\sum_{j} Y\left(t_{j-1}\right)^{2}\left(W\left(t_{j}\right)-W\left(t_{j-1}\right)\right)^{2} \rightarrow \int_{0}^{t} Y(s)^{2} d s
$$


on $\Omega^{\prime}$ by the convergence given in Lemma 4.2. Finally

$$
\begin{aligned}
& \sum_{j}\left(Y\left(t_{j}\right) W\left(t_{j}\right)-Y\left(t_{j-1}\right) W\left(t_{j-1}\right)\right)\left(\bar{Y}\left(t_{j}\right) \bar{W}\left(t_{j}\right)-\bar{Y}\left(t_{j-1}\right) \bar{W}\left(t_{j-1}\right)\right) \\
& =\sum_{j}\left[Y\left(t_{j-1}\right)\left(W\left(t_{j}\right)-W\left(t_{j-1}\right)\right)+\left(Y\left(t_{j}\right)-Y\left(t_{j-1}\right)\right) W\left(t_{j}\right)\right] \\
& \times\left[\bar{Y}\left(t_{j-1}\right)\left(\bar{W}\left(t_{j}\right)-\bar{W}\left(t_{j-1}\right)\right)+\left(\bar{Y}\left(t_{j}\right)-\bar{Y}\left(t_{j-1}\right)\right) \bar{W}\left(t_{j}\right)\right] \\
& =\sum_{j} Y\left(t_{j-1}\right) \bar{Y}\left(t_{j-1}\right)\left(W\left(t_{j}\right)-W\left(t_{j-1}\right)\right)\left(\bar{W}\left(t_{j}\right)-\bar{W}\left(t_{j-1}\right)\right)+\varepsilon_{n},
\end{aligned}
$$

where $\varepsilon_{n} \rightarrow 0$ a.s., as $n \rightarrow \infty$. Again by Lemma 4.3 the sum tends to zero on $\Omega^{\prime}$.

Proof of Lemma 4.2 For any measure, the fact that $\mu_{n} \Rightarrow \mu$ follows from $\mu_{n}([0, s]) \rightarrow$ $\mu([0, s])$ for all $s \in[0, t], s$ rational. From any subsequence of the given sequence, one can extract a further subsequence such that $\mu_{n}([0, s]) \rightarrow \mu([0, s])$, for all $s$ rational, $0 \leq s \leq t$, a.s. Hence along that subsequence $\mu_{n} \Rightarrow \mu$ a.s., hence the whole sequence converges weakly in probability.

Proof of Lemma 4.3 Write $\Delta_{j} W$ for $W\left(s_{j}^{n}\right)-W\left(s_{j-1}^{n}\right)$ and $\Delta_{j} \bar{W}$ for $\bar{W}\left(s_{j}^{n}\right)-\bar{W}\left(s_{j-1}^{n}\right)$. Note that for all $0 \leq r<s \leq t$, as $n \rightarrow \infty$,

$$
\sum_{r<s_{j}^{n} \leq s} \Delta_{j} W \Delta_{j} \bar{W} \rightarrow 0 \text { in probability, as } n \rightarrow \infty .
$$

Consequently if $f$ is a step function,

$$
\sum_{j=1}^{n} \Delta_{j} W \Delta_{j} \bar{W} f\left(s_{j-1}^{n}\right) \rightarrow 0 \text { in probability, as } n \rightarrow \infty .
$$

Moreover for any two functions $f$ and $g$

$$
\begin{aligned}
& \left|\sum_{j=1}^{n} \Delta_{j} W \Delta_{j} \bar{W}\left(f\left(s_{j-1}^{n}\right)-g\left(s_{j-1}^{n}\right)\right)\right| \\
& \leq \sup _{0 \leq s \leq t}|f(s)-g(s)|\left(\sum_{j=1}^{n}\left(\Delta_{j} W\right)^{2}\right)^{\frac{1}{2}}\left(\sum_{j-=1}^{n}\left(\Delta_{j} \bar{W}\right)^{2}\right)^{\frac{1}{2}},
\end{aligned}
$$

and the right hand side tends to

$$
t \times \sup _{0 \leq s \leq t}|f(s)-g(s)|
$$

in probability, as $n \rightarrow \infty$.

Let now $f$ be a continuous function, and $g$ be a step function. Choose

$$
\delta=2 t \sup _{0 \leq s \leq t}|f(s)-g(s)|
$$


We have

$$
\begin{aligned}
\mathbb{P}\left\{\left|\sum f\left(s_{j-1}^{n}\right) \Delta_{j} W \Delta_{j} \bar{W}\right|>\right. & \delta\} \leq \mathbb{P}\left\{\left|\sum_{j=1}^{n} g\left(s_{j-1}^{n}\right) \Delta_{j} W \Delta_{j} \bar{W}\right|>\delta / 3\right\} \\
& +\mathbb{P}\left\{\left|\sum_{j=1}^{n}\left(f\left(s_{j-1}^{n}\right)-g\left(s_{j-1}^{n}\right)\right) \Delta_{j} W \Delta_{j} \bar{W}\right|>2 \delta / 3\right\},
\end{aligned}
$$

and it follows from the above arguments that the latter tends to zero as $n \rightarrow \infty$. Since $\delta$ can be made arbitrarily small by an appropriate choice of the step function $g$, the lemma is proved.

\section{Relation to Brackets of vector fields}

We now sketch another possible proof of our Theorem 3.1 which brings in explicitly the brackets of certain vector fields. A vector field over the space $\mathbb{L}^{2}$ is a mapping from a dense subset of $\mathbb{L}^{2}$ into itself. We begin by rewriting 1.1 as

$$
\frac{\partial w}{\partial t}(t)=F_{0}(w(t))+\sum_{i=1}^{N} F_{i} \frac{\partial W_{i}}{\partial t}(t) .
$$

The diffusion vector fields in our case are constant vector fields defined by

$$
F_{i}=e_{k_{i}}, \quad 1 \leq i \leq N,
$$

where $N$ is the cardinality of $\mathscr{Z}_{*}$ and $\left\{k_{1}, \cdots, k_{N}\right\}$ is any ordering of the set $\mathscr{Z}_{*}$. Similarly the drift vector field is denoted by $F_{0}(w)=v \Delta w-B(w, w)$. In this notation, 2.2, becomes

$$
\left\{\begin{array}{l}
\frac{\partial U^{t, \phi}}{\partial s}(s)+\left(\nabla_{w} F_{0}\right)^{*}(w(s)) U^{t, \phi}(s)=0, \quad 0 \leq s \leq t ; \\
U^{t, \phi}(t)=\phi,
\end{array}\right.
$$

where $\nabla_{w} F_{0}$ is the Fréchet derivative of $F_{0}$ in the $\mathbb{L}^{2}$ topology and $\left(\nabla_{x} F_{0}\right)^{*}$ is its $\mathbb{L}^{2}$-adjoint. If it is well defined, we define the bracket $[F, G]$ between two $\mathbb{L}^{2}$ vector fields $F$ and $G$ as $[F, G]=\left(\nabla_{w} F\right) G-\left(\nabla_{w} G\right) F$. (Part of being well defined is that the range of $G$ and $F$ are contained in the domain of $\nabla_{w} F$ and $\nabla_{w} G$ respectively.)

The argument in this alternate proof is based on the two next results.

Lemma 5.1. Let $G$ be a vector field on $\mathbb{L}^{2}$ which is twice Fréchet differentiable in the $\mathbb{L}^{2}$ topology and such that $\left[F_{i}, G\right], i=0 \ldots N$, are vector fields on $\mathbb{L}^{2}$. Then we have

$$
\begin{aligned}
& \left\langle U^{t, \phi}(s), G(w(s))\right\rangle-\left\langle U^{t, \phi}(0), G(w(0))\right\rangle \\
& =\int_{0}^{s}\left\langle U^{t, \phi}(r),\left[F_{0}, G\right](w(r))\right\rangle d r+\sum_{i=1}^{N} \int_{0}^{s}\left\langle U^{t, \phi}(r),\left[F_{i}, G\right](w(r))\right\rangle \circ d W_{r}^{i}
\end{aligned}
$$


where o means that it is a Stratonovich (anticipating) integral; in Itô-Skorohod language, it takes the form

$$
\begin{aligned}
& =\int_{0}^{s}\left\langle U^{t, \phi}(r),\left[F_{0}, G\right](w(r))\right\rangle d r+\sum_{i=1}^{N} \int_{0}^{s}\left\langle U^{t, \phi}(r),\left[F_{i}, G\right](w(r))\right\rangle d W_{r}^{i} \\
& +\sum_{i=1}^{N} \int_{0}^{s}\left[\frac{1}{2}\left\langle\nabla_{w}^{2} G(w(r))\left(F_{i}, F_{i}\right), U^{t, \phi}(r)\right\rangle+\left\langle\left[F_{i}, G\right](w(r)), D_{r}^{i} U^{t, \phi}(r)\right\rangle\right] d r
\end{aligned}
$$

Proof. The formula in Skorohod language follows from Theorem 6.1 in [NP88], via an easy finite dimensional approximation. Its translation in the Stratonovich form follows from Theorem 7.3 in the same paper (see also Theorem 3.1.1 in [Nua95).

We can now prove the following:

Proposition 5.2. Let $\Omega_{0} \subset \Omega$. Under the same assumptions on $G$ as in the above Lemma,

$$
\left\langle U^{t, \phi}(s), G(w(s))\right\rangle \equiv 0 \text { on the set } \Omega_{0}
$$

implies that

$$
\left\langle U^{t, \phi}(s),\left[F_{i}, G\right](w(s))\right\rangle \equiv 0 \text { a.s. on the set } \Omega_{0}, i=0,1, \ldots, N .
$$

Proof. The assumption implies that the quadratic variation on $[0, t]$ of the process $\left\{\left\langle U^{t, \phi}(s), G(w(s))\right\rangle\right\}$ vanishes almost surely on $\Omega_{0}$. Then from [Nua95], Theorem 3.2.1, for $i=1, \ldots, N$,

$$
\int_{0}^{t}\left\langle U^{t, \phi}(s),\left[F_{i}, G\right](w(s))\right\rangle^{2} d s=0
$$

a. s. on the set $\Omega_{0}$, i.e.

$$
\left\langle U^{t, \phi}(s),\left[F_{i}, G\right](w(s))\right\rangle \equiv 0 \text { a. s. on the set } \Omega_{0}, i=1, \ldots, N .
$$

This implies that for $1 \leq i \leq N$,

$$
\int_{0}^{s}\left\langle U^{t, \phi}(r),\left[F_{i}, G\right](w(r))\right\rangle \circ d W_{r}^{i} \equiv 0 \text { a. s. on the set } \Omega_{0}
$$

(see Definition 3.1.1 in [Nua95]), from which it follows (see the previous Lemma) that

$$
\left\langle U^{t, \phi}(s),\left[F_{0}, G\right](w(s))\right\rangle \equiv 0 \text { a. s. on the set } \Omega_{0} .
$$

Now call $\mathscr{L}$ all well defined $\mathbb{L}^{2}$ vector fields in the ideal generated by the vector fields $F_{1}, \ldots, F_{N}$ in the Lie algebra generated by $F_{0}, F_{1}, \ldots, F_{N}$. In other words, at each $u \in \mathbb{L}^{2}, \mathscr{L}(u)$ consists of $F_{1}, \ldots, F_{N}$, and all brackets

$$
\left[F_{i_{n}},\left[F_{i_{n-1}}, \ldots\left[F_{i_{2}}, F_{i_{1}}\right] \ldots\right](u)\right. \text {, }
$$


which are well defined vector fields on $\mathbb{L}^{2}$ where $1 \leq i_{1} \leq N$, and for $j>1,0 \leq$ $i_{j} \leq N$. Iterating the argument in the Proposition, we deduce the following result.

Corollary 5.3. Given $\phi \in \mathbb{L}^{2}$, let

$$
\Omega_{0}:=\{\langle\mathscr{M}(t) \phi, \phi\rangle=0\} .
$$

If $\mathbb{P}\left(\Omega_{0}\right)>0$, then

$$
\langle\phi, G(w(s))\rangle \equiv 0, \quad \forall G \in \mathscr{L}, \text { a. s. on } \Omega_{0} .
$$

In particular, $\phi$ is orthogonal to all constant vector fields in $\mathscr{L}$.

In the case of the stochastic Navier Stokes equations given in (1.1), all of the brackets given in (5.1) are well defined if the $e_{k}$ used to define the forcing have exponentially decaying Fourier components. This follows from the fact that all of the bracket of the form (5.1) contain differential operators with polynomial symbols and the fact that, with this type of forcing, on any finite time interval $[0, T]$ there exists a positive random variable $\gamma$ so that $\sup _{[0, T]}\left\|e^{\gamma|\nabla|} w(s)\right\|^{2}<\infty$ almost surely. Here $\left\|e^{\gamma|\nabla|} w\right\|^{2}=\sum_{k} e^{2 \gamma|k|}\left|w_{k}\right|^{2}$ where $w(t, x)=\sum_{k} w_{k}(t) e^{i k \cdot x}$. See for example [Mat02] for a stronger version of this result or [MS99 Mat98] for simpler versions.

Furthermore in [EM01] it was implicitly shown by the construction used that the span of the constant vector fields contains $S_{\infty}$. Thus, under the same conditions as before we see that the law of arbitrary finite dimensional projections of $w(t)$ have a density with respect to Lebesgue measure.

\section{Smoothness}

In the preceding sections, we proved the existence of a density. We now address the smoothness of the density. While the former simply required that the projected Malliavin matrix be invertible, the proof of smoothness requires control on the norm of the inverse of the projected Malliavin matrix together with "smoothness in the Malliavin sense." The following is the main result of this section; however, it rests heavily on the general results proven in Section 7 as well as some technical results from the appendices.

Theorem 6.1. Let $S$ be any finite dimensional subspace of $S_{\infty}$ and $\Pi$ the orthogonal projection in $\mathbb{L}^{2}$ onto $S$. For any $t>0$, the law of $\Pi w_{t}$ has a $C^{\infty}$ density with respect to the Lebesgue measure on $S$.

Proof. We use Corollary 2.1.2 of [Nua95]. Lemma[C.1 from the appendix establishes condition (i) from that corollary while condition (ii) of the same corollary follows from the next theorem.

The following is a quantitative version of Proposition 3.5 It gives a quantitative control of the smallest eigenvalue of a finite dimensional projection of the Malliavin matrix. 
Theorem 6.2. Let $\Pi$ be the orthogonal projection of $\mathbb{L}^{2}$ onto a finite dimensional subspace of $S_{\infty}$. For any $T>0, \eta>0, p \geq 1$, and $K>0$ there exists a constant $c=c\left(v, \eta, p,\left|\mathscr{Z}_{*}\right|, T, K, \Pi\right)$ and $\varepsilon_{0}=\varepsilon_{0}\left(v, K,\left|\mathscr{Z}_{*}\right|, T, \Pi\right)$ so that for all $\varepsilon \in\left(0, \varepsilon_{0}\right]$,

$$
\mathbb{P}\left(\inf _{\phi \in S(K, \Pi)}\langle\mathscr{M}(T) \phi, \phi\rangle<\varepsilon\right) \leq c \exp \left(\eta\|w(0)\|^{2}\right) \varepsilon^{p}
$$

where $S(K, \Pi)=\left\{\phi \in S_{\infty}:\|\phi\|_{1} \leq 1,\|\Pi \phi\| \geq K\right\}$.

Remark 6.3. Notice that this lemma implies that the eigenvectors with "small" eigenvalues have small projections in the "lower" modes. The definition of "lower" modes depends on the definition of "small" eigenvalues. This separation between the eigenvectors with small eigenvalues and the low modes is one of the keys to the ergodic results proved in [HM04].

Remark 6.4. Also notice that there is a mismatch in the topology in Theorem 6.2 in that the test functions are bounded in $\mathbb{H}^{1}$ but the innerproduct is in $\mathbb{L}^{2}$. This can likely be rectified since the backward adjoint linearized flow $\bar{J}_{s, T}^{*}$ maps $\mathbb{L}^{2}$ into $\mathbb{H}^{1}$ for any $s \leq T$, it is possible to obtain estimates on $\mathbb{P}(\langle\phi, \mathscr{M}(T) \phi\rangle<\varepsilon)$ for $\phi \in \mathbb{L}^{2}$. Just as in the proof of Theorem 6.2 where we exclude a small neighborhood of time zero to allow $w_{t}$ to regularize, we could exclude the $s$ in a small neighborhood of the terminal $T$ to allow $U^{T, \phi}(s)=J_{s, T}^{*} \phi$ to regularize.

Proof. Recall the definition of $S_{n}$ and $\mathscr{Z}_{n}$ from $(3.1)$ and let $\Pi_{n}$ be the orthonormal projection onto $S_{n}$. Since $S(K, \Pi) \subset S_{\infty}$ and $\Pi$ projects onto a finite dimensional subspace of $S_{\infty}$, for $n$ sufficiently large $\left\|\Pi_{n} \phi\right\|>\frac{1}{2} K$ for all $\phi \in S(K, \Pi)$. Fix such an $n$.

We now construct a basis of $S_{n}$ compatible with the structure of $\mathscr{Z}_{k}, k \leq n$. Fixing any ordering of $\mathscr{Z}_{*}$, set $\left\{f_{i}: i=1, \ldots, N=\left|\mathscr{Z}_{*}\right|\right\}=\mathscr{Z}_{*}$. Clearly $\left\{f_{i}\right\}_{i=1}^{N}$ is a basis for $S_{0}$. Set $J_{0}=N$. By the construction of $S_{n}$ it is clear that $S_{n} \backslash S_{n-1}$ is equal to the $\operatorname{span}\left(e_{k}: k \in \mathscr{Z}_{n}^{\prime}\right)$ where $\mathscr{Z}_{n}^{\prime} \stackrel{\text { def }}{=} \mathscr{Z}_{n} \cap_{j=0}^{n-1} \mathscr{Z}_{j}^{c} \cap \mathscr{Z}_{*}^{c}$. For $n \geq 1$, set $J_{n}=J_{n-1}+\left|\mathscr{Z}_{n}^{\prime}\right|$ and $\left\{f_{i}: i=J_{n-1}+1, \ldots, J_{n}\right\}=\left\{e_{k}: k \in \mathscr{Z}_{n}^{\prime}\right\}$, again fixing an arbitrary ordering of the righthand side. Clearly $\left\{f_{i}\right\}_{i=1}^{J_{n}}$ chosen in this way is an orthogonal basis for $S_{n}$.

Fix some $t_{0} \in(0, T)$. Recall that by Corollary 2.4

$$
\mathbb{P}\left(\inf _{\phi \in S(K, \Pi)}\langle\mathscr{M}(T) \phi, \phi\rangle<\varepsilon\right)=\mathbb{P}\left(\inf _{\phi \in S(K, \Pi)} \sum_{k \in \mathscr{Z}_{*}} \int_{0}^{T}\left\langle U^{T, \phi}(s), e_{k}\right\rangle^{2} d s<\varepsilon\right) .
$$

Let $X^{\phi}$ and $Y_{j}^{\phi}$ be as in proposition 3.7 For $j=1, \cdots, J_{n}$, define

$$
\chi_{j}^{\phi}(t)=-\left\langle X^{\phi}(T-t)+\sum_{k \in \mathscr{Z}_{*}} Y_{k}^{\phi}(T-t) W_{k}(T), f_{j}\right\rangle,
$$


$\Upsilon_{j, k}^{\phi}(t)=\left\langle Y_{k}^{\phi}(T-t), f_{j}\right\rangle, G_{j}^{\phi}(t)=\left\langle U^{T, \phi}(T-t), f_{j}\right\rangle$, and $\bar{W}_{k}(t)=W_{k}(T)-W_{k}(T-$ $t)$. Notice that for $t \in[0, T]$ and $j=1, \cdots, J_{n}$

$$
G_{j}^{\phi}(t)=\left\langle\phi, f_{j}\right\rangle+\int_{0}^{t}\left[\chi_{j}^{\phi}(s)+\sum_{k \in \mathscr{Z}_{*}} \Upsilon_{j, k}^{\phi}(s) \bar{W}_{k}(s)\right] d s .
$$

Furthermore in light of the observations in the proof of Lemma 3.6. we see that this sequence of equations satisfies the assumptions of the next subsection with $J_{0}$ as defined above and $J=J_{n}$. Next recall that $U^{t, \phi}(s, x)=\sum_{k \in \mathbb{Z}_{0}^{2}} \beta_{k}^{\phi}(s) e_{k}(x)$. Combining this, the last equalities in Proposition 3.7 and the argument already used at the end of the proof of Lemma 3.6 we see that each $G_{j}^{\phi}$ is a linear combination of the $\left\{\Upsilon_{i, k}^{\phi}: i<j, k \in \mathscr{Z}_{*}\right\}$ with coefficients which are constant in time.

Below $\|\cdot\|_{1,[a, b]}$ and $\|\cdot\|_{\infty,[a, b]}$ respectively denote the Lipschitz and $L^{\infty}$ norm on $[a, b]$, see the beginning of section 7 for the precise definitions.

Fix a $t \in(0, T)$ and set $T_{1}=T-t$. Bounds, uniform for $\phi \in S(K, \Pi)$, on the $p$-th moments of the $L^{\infty}$-norm and Lipschitz constants of $\chi_{j}^{\phi}$, and $\Upsilon_{j, k}^{\phi}$ over the interval $\left[0, T_{1}\right]$ are given by Lemma E.2 (Recall that these processes have been time reversed.) Hence given any $p \geq 1, q>0$ and $\eta>0$, there exists a $c=c\left(\eta, q, p, t, v, \mathscr{E}_{1}, T\right)>0$ so that for any $\varepsilon>0$ if one defines

$$
\Omega_{b}(\varepsilon, q)=\bigcap_{\phi \in S(K, \Pi)}\left\{\sup _{k, j}\left(\left\|\chi_{j}^{\phi}\right\|\left\|_{1,\left[0, T_{1}\right]},\right\| \Upsilon_{j, k}^{\phi} \|_{1,\left[0, T_{1}\right]}\right) \leq \varepsilon^{-q}\right\}
$$

then the estimate $\mathbb{P}\left(\Omega_{b}(\varepsilon, q)^{c}\right) \leq c \exp \left(\eta\|w(0)\|^{2}\right) \varepsilon^{p}$ holds.

Next Corollary 7.3 and Proposition 7.1 state that there exist $q=q(|S|, N)$ and $\varepsilon_{0}=\varepsilon_{0}\left(T, \mathscr{Z}_{*}|| S \mid,\right)$ so that for all $\varepsilon \in\left(0, \varepsilon_{0}\right]$ there is a $\Omega_{\sharp}(\varepsilon)$ so that for all $\phi \in$ $S(K, \Pi)$ one has

$$
\left\{\sup _{i=1, \cdots, N} \int_{0}^{T_{1}}\left|G_{i}^{\phi}(s)\right|^{2} d s<\varepsilon ; \sup _{i=1, \cdots, J_{n}} \sup _{s \in\left[0, T_{1}\right]}\left|G_{i}^{\phi}(s)\right|>\varepsilon^{q}\right\} \cap \Omega_{b}(\varepsilon, q) \subset \Omega_{\sharp}(\varepsilon)
$$

and $\mathbb{P}\left(\Omega_{\sharp}(\varepsilon)\right) \leq c \varepsilon^{p}$ for all $p \geq 1$ and $\eta>0$ with a $c=c\left(T,\left|\mathscr{Z}_{*}\right|,|S|, p, \eta, v\right)$. Notice that because of the uniformity in 6.1), $\Omega_{\sharp}(\varepsilon)$ does not depend on the sequence of $G$ 's. Since $\sup _{k \in \mathscr{Z}_{*}} \int_{t}^{T}\left\langle U^{T, \phi}(s), e_{k}\right\rangle^{2} d s=\sup _{i=1, \cdots, N} \int_{0}^{T_{1}}\left|G_{i}^{\phi}(s)\right|^{2} d s$ and $\sup _{s \in\left[0, T_{1}\right]}\left|G_{i}^{\phi}(s)\right|=\left\|\left\langle U^{T, \phi}, f_{i}\right\rangle\right\|_{\infty,[t, T]}$, the inclusion given in (6.2] becomes

$\left\{\sup _{k \in \mathscr{Z}_{*}} \int_{t}^{T}\left\langle U^{T, \phi}(s), e_{k}\right\rangle^{2} d s<\varepsilon ; \sup _{i=1, \cdots, J_{n}}\left\|\left\langle U^{T, \phi}, f_{i}\right\rangle\right\|_{\infty,[t, T]}>\varepsilon^{q}\right\} \subset \Omega_{\sharp}(\varepsilon) \cup \Omega_{b}(\varepsilon, q)^{c}$

for all $\varepsilon \in\left(0, \varepsilon_{0}\right]$. Since $\left\langle U^{T, \phi}(T), f_{i}\right\rangle=\left\langle\phi, f_{i}\right\rangle$, by the choice of the subspace $S_{n}$ one has $\sup _{i}\left\|\left\langle U^{T, \phi}, f_{i}\right\rangle\right\|_{\infty,[t, T]} \geq \frac{K}{2 \sqrt{J_{n}}}$. Thus for $\varepsilon \in\left(0, \varepsilon_{0} \wedge\left(\frac{K}{2 \sqrt{J_{n}}}\right)^{\frac{1}{q}}\right]$ one has 
$\varepsilon^{q} \leq \frac{K}{2 \sqrt{J_{n}}}$ which transforms $\underline{6.3}$ into

$$
\left\{\sum_{k \in \mathscr{Z}_{*}} \int_{t}^{T}\left\langle U^{T, \phi}(s), e_{k}\right\rangle^{2} d s<\varepsilon\right\} \subset \Omega_{\sharp}(\varepsilon) \cup \Omega_{b}(\varepsilon, q)^{c} .
$$

As $\phi$ was an arbitrary direction in $S(K, \Pi)$ and $\Omega_{\sharp}(\varepsilon)$ and $\Omega_{b}(\varepsilon, q)$ are independent of $\phi$, we have

$$
\begin{aligned}
\left\{\inf _{\phi \in S(K, \Pi)} \sum_{k \in \mathscr{Z}_{*}} \int_{t}^{T}\left\langle U^{T, \phi}(s), e_{k}\right\rangle^{2} d s<\varepsilon\right\}= \\
\bigcup_{\phi \in S(K, \Pi)}\left\{\sum_{k \in \mathscr{Z}_{*}} \int_{t}^{T}\left\langle U^{T, \phi}(s), e_{k}\right\rangle^{2} d s<\varepsilon\right\} \subset \Omega_{\sharp}(\varepsilon) \cup \Omega_{b}(\varepsilon, q)^{c} .
\end{aligned}
$$

In summary we have shown that for any $p \geq 1$ and $\eta>0$, there exists $q>0$ so that the above inclusion holds and a $c>0$ so that $\mathbb{P}\left(\Omega_{\sharp}(\varepsilon)\right)+\mathbb{P}\left(\Omega_{b}(\varepsilon, q)^{c}\right) \leq$ $c \exp \left(\eta\|w(0)\|^{2}\right) \varepsilon^{p}$.

\section{Controlling the Chance of Being Small}

This section contains the main estimate used to control the chance of certain processes being small when their quadratic variation is large. The estimates of this section are simply quantitative versions of the results of Section 4 . There are also the analog of results used in the standard Malliavin calculus as applied to finite dimensional SDEs. There the estimates were developed by Stroock [Str83] and Norris [Nor86]. Here we do not have adapted processes. Instead, we exploit the smoothness in time to obtain estimates.

For the entirety of this section, we fix a time $T$ and consider only the interval of time $[0, T]$. For any real-valued function of time $f$, define the $\alpha$-Hölder constant over the time interval $[0, T]$ by

$$
\mathscr{H}_{\alpha}(f)=\sup _{\substack{s, r \in[0, T] \\ 0<|s-r| \leq 1}} \frac{|f(s)-f(r)|}{|s-r|^{\alpha}}
$$

and the $L^{\infty}$ norm by

$$
\|f\|_{\infty}=\sup _{s \in[0, T]}|f(s)| .
$$

We also define $\|f\|_{\alpha}=\max \left(\|f\|_{\infty}, \mathscr{H}_{\alpha}(f)\right)$. At times we will also need versions of the above norms over shorter intervals of time. For $[a, b] \subset[0, T]$ we will write $\mathscr{H}_{\alpha,[a, b]}(f),\|f\|_{\infty,[a, b]}$, and $\|f\|_{\alpha,[a, b]}$ for the norms with the same definitions as above except that the supremum over $[0, T]$ is replaced with a supremum over $[a, b]$. We also extend the definitions of the Lipschitz constant in time $\mathscr{H}_{\alpha}(f)$, to functions of time talking values in $\mathbb{L}^{2}$ by replacing the absolute value in the definitions given in (7.1) and (7.2) by the norm on $\mathbb{L}^{2}$. Similarly we extend the definition $\mathscr{H}_{\alpha,[a, b]}(f)$, $\|f\|_{\infty},\|f\|_{\infty,[a, b]}$ to functions of time taking values in $\mathbb{L}^{2}$. 


\subsection{A Ladder of Estimates}

For each $1 \leq j \leq J$, let $\left\{W_{i}^{(j)}: i=1 \ldots N\right\}$ be a collection of mutually independent standard Wiener processes with $W_{i}(0)=0$ defined on a probability space $(\Omega, \mathscr{F}, \mathbb{P})$.

We say that the collection of processes $\mathscr{G}=\left\{G^{(j)}(t, \omega): 1 \leq j \leq J\right\}$ forms a ladder of order $J$ with base size $J_{0}$ if first $1 \leq J_{0}<J$ and

$$
\begin{aligned}
G^{(j)}(t, \omega) & =G_{0}^{(j)}+\int_{0}^{t} H^{(j)}(s, \omega) d s \\
H^{(j)}(s, \omega) & =X^{(j)}(s, \omega)+\sum_{i=1}^{N} Y_{i}^{(j)}(s) W_{i}^{(j)}(s, \omega)
\end{aligned}
$$

where $j=1, \ldots, J<\infty$ and $\omega \in \Omega$.

Second, we require that for $j$ greater than $J_{0}$, the $G^{(j)}$ are determined by the functions at the previous levels. More precisely, for each $j$ with $j>J_{0}$ there exists an integer $K=K(j)$, a collection $\left\{g_{k}(t): k=1, \ldots, K\right\}$ of bounded, deterministic functions of time, and a collection $\left\{f_{k}(t, \omega): k=1, \ldots, K\right\}$ of stochastic process with

$$
f_{k} \in\left\{Y_{i}^{(l)}, X^{(l)}, G^{(n)}: 1 \leq i \leq N, 1 \leq l \leq j-1,1 \leq n \leq j-1\right\}
$$

so that

$$
G^{(j)}(t, \omega)=\sum_{k=1}^{K} g_{k}(t) f_{k}(t, \omega) \text { almost surely. }
$$

This assumption can be restated by saying that, for $j>J_{0}, G^{(j)}$ must be at each moment of time in the span of the preceding $X, Y$, and $G$. And furthermore, the coefficients in the linear combination producing $G^{(j)}$ must be uniformly bounded on $[0, T]$.

It is important to remark that we do not assume that the $Y_{i}^{(j)}$ or $X^{(j)}$ are adapted to the Wiener processes. Typical assumptions regarding adaptedness will be replaced with assumptions on the regularity of the processes in time.

The goal of this section is to prove that under certain assumptions, if the first $J_{0}$ of the $G^{(j)}$ are small in some sense then all of the $X, Y$, and remaining $G$ are also small with high probability. The ladder structure connects the $j$-th level with the other levels.

Fix a time $T>0$. For any choice of the positive parameter $\Delta$ define $\delta=\Delta^{\frac{5}{3}}$. For $k=0,1, \ldots$, define $t_{k}=k \Delta \wedge T$. For each fixed $k$, define $s_{\ell}(k)=\left(t_{k}+\ell \delta\right) \wedge t_{k+1}$ for $\ell=0,1, \ldots$ Set $\delta_{\ell}^{k}=s_{\ell}(k)-s_{\ell-1}(k)$ and $\delta_{\ell}^{k} f=f\left(s_{\ell}(k)\right)-f\left(s_{\ell-1}(k)\right)$. Lastly define $m=\inf \left\{k: t_{k}=T\right\}$ and $M(k)=\inf \left\{\ell: s_{\ell}(k)=t_{k+1}\right\}$. Notice that $\Delta^{-\frac{2}{3}}=$ $\frac{\Delta}{\delta} \leq M(k) \leq \frac{\Delta}{\delta}+1=\Delta^{-\frac{2}{3}}+1$ for all $k$ and $m \leq T \Delta^{-1}+1$. 
Define the following subsets of the probability space $\Omega$ :

$$
\begin{aligned}
& \Omega_{a}(\Delta)=\left\{\inf _{0 \leq k \leq m} \inf _{1 \leq i \leq N} \frac{1}{M(k)} \sum_{\ell=1}^{M(k)} \frac{\left(\delta_{\ell}^{k} W_{i}\right)^{2}}{\delta_{\ell}^{k}} \leq \frac{1}{2}\right\} \\
& \Omega_{b}(\Delta)=\left\{\sup _{0 \leq k \leq m} \sup _{\substack{(i, j) \in\{1, \ldots, N\}^{2} \\
i \neq j}} \frac{1}{M(k)}\left|\sum_{\ell=1}^{M(k)} \frac{\left(\delta_{\ell}^{k} W_{i}\right)\left(\delta_{\ell}^{k} W_{j}\right)}{\delta_{\ell}^{k}}\right| \geq \frac{\Delta^{\frac{3}{14}}}{3 N^{2}}\right\} \\
& \Omega_{c}(\Delta)=\left\{\sup _{1 \leq i \leq N}\left\|W_{i}\right\|_{\frac{1}{4}}>\Delta^{-\frac{1}{28}}\right\}, \\
& \Omega_{\sharp}^{\prime}(\varepsilon)=\Omega_{a}\left(\varepsilon^{\frac{14}{75}}\right) \cup \Omega_{b}\left(\varepsilon^{\frac{14}{75}}\right) \cup \Omega_{c}\left(\varepsilon^{\frac{14}{75}}\right) \text { and finally } \\
& \Omega_{\sharp}(\varepsilon)=\bigcup_{j=1}^{J} \Omega_{\sharp}^{\prime}\left(\varepsilon^{\left(\frac{1}{152}\right)^{j}}\right) .
\end{aligned}
$$

The following bound follows readily from Corollary 7.11 and Lemma 7.12 in section 7.4

Proposition 7.1. For any $p \geq 1$, there exists a constant $c=c(T, J, N, p)$, in particular independent of $\varepsilon$, such that

$$
\mathbb{P}\left(\Omega_{\sharp}(\varepsilon)\right) \leq c \varepsilon^{p} .
$$

The next key result in this section is the following proposition which shows why the previous estimate is important.

Proposition 7.2. Fix a positive integer $J$ and for $q>0$ define

$$
\begin{aligned}
& \Omega_{q *}(\mathscr{G}, \varepsilon)=\left\{\sup _{\substack{1 \leq j \leq J \\
1 \leq i \leq N}}\left(\left\|X^{(j)}\right\|_{1},\left\|Y_{i}^{(j)}\right\|_{1}\right) \leq \varepsilon^{-q}\right\}, \\
& \Omega_{q}(\mathscr{G}, \varepsilon)=\left\{\sup _{1 \leq j \leq J_{0}}\left\|G^{(j)}\right\|_{\infty}<\varepsilon ; \sup _{\substack{1 \leq j \leq J \\
1 \leq i \leq N}}\left(\left\|X^{(j)}\right\|_{\infty},\left\|Y_{i}^{(j)}\right\|_{\infty},\left\|G^{(j)}\right\|_{\infty}\right)>\varepsilon^{q}\right\} .
\end{aligned}
$$

Then there exists positive constants $q_{0}=q_{0}(J)$ and $\varepsilon_{0}=\varepsilon_{0}(T, J, N)$ so that for any $\varepsilon \in\left(0, \varepsilon_{0}\right], q \in\left(0, q_{0}\right]$ and ladder $\mathscr{G}=\left\{G^{(j)}: 1 \leq j \leq J\right\}$ of order $J$ with base size $J_{0}$ less than $J$,

$$
\Omega_{q *}(\mathscr{G}, \varepsilon) \cap \Omega_{q}(\mathscr{G}, \varepsilon) \subset \Omega_{\sharp}(\varepsilon) .
$$

In words, this proposition states that if the first $J_{0}$ of the $G^{(j)}$ are small and all of the quantities $\left\|X^{(j)} \mid\right\|_{1},\left\|Y_{i}^{(j)}\right\|_{1}$ are not to big then it is unlikely that the remaining $G^{(j)}$ are big. 
Proof of Proposition 7.2 The idea of the proof is to iterate Lemma 7.5 below. Begin by setting $g_{*}=\sup _{j} \sum_{k=1}^{K(j)}\left|g_{k}\right|_{\infty}$. Now define $\varepsilon_{0}=\tilde{\varepsilon}_{0}=\varepsilon$ and for $j>1$ define $\varepsilon_{j}=\tilde{\varepsilon}_{j-1}^{\frac{1}{15}}, \tilde{\varepsilon}_{j}=\varepsilon_{j}^{\frac{151}{152}}$. With these choices, $\varepsilon_{j}<\tilde{\varepsilon}_{j}<\varepsilon_{j+1}<\tilde{\varepsilon}_{j+1}$. We also choose $q_{0}$ sufficiently small so that $\tilde{\varepsilon}_{J+1}<\varepsilon^{q}$ for $\varepsilon \in[0,1)$ and $q \in\left(0, q_{0}\right]$. Define the following subsets of $\Omega$

$$
\begin{aligned}
A(j) & =\Omega_{q *}(\mathscr{G}, \varepsilon) \cap \Omega_{q}(\mathscr{G}, \varepsilon) \cap\left\{\sup _{1 \leq i \leq N}\left(\left\|X^{(l)}\right\|_{\infty},\left\|Y_{i}^{(l)}\right\|_{\infty}\right) \leq \varepsilon_{l+1} \text { for } l \leq j\right\} \\
A^{+}(j) & =A(j) \cap\left\{\left\|G^{(l)}\right\|_{\infty} \leq \tilde{\varepsilon}_{l} \leq \varepsilon_{l+1} \text { for } l \leq j+1\right\} \\
B(j) & =\left\{\sup _{1 \leq i \leq N}\left(\left\|X^{(j)}\right\|_{\infty},\left\|Y_{i}^{(j)}\right\|_{\infty}\right)>\tilde{\varepsilon}_{j}^{\frac{1}{151}}=\varepsilon_{j+1}\right\} \\
C(j) & =\Omega_{q *}(\mathscr{G}, \varepsilon) \cap\left\{\left\|G^{(j)}\right\|_{\infty} \leq \tilde{\varepsilon}_{j} ; \sup _{1 \leq i \leq N}\left(\left\|X^{(j)}\right\|_{\infty},\left\|Y_{i}^{(j)}\right\|_{\infty}\right)>\tilde{\varepsilon}_{j}^{\frac{1}{151}}=\varepsilon_{j+1}\right\} .
\end{aligned}
$$

First notice that since $\varepsilon<\varepsilon^{q}$ for $\varepsilon \in(0,1]$ and $q \in\left(0, q_{0}\right]$, the event

$$
\left\{\sup _{1 \leq j \leq J_{0}}\left\|G^{(j)}\right\|_{\infty}<\varepsilon ; \sup _{1 \leq j \leq J_{0}}\left\|G^{(j)}\right\|_{\infty}>\varepsilon^{q}\right\}
$$

is empty. Hence

$$
\Omega_{q}(\mathscr{G}, \varepsilon)=\left\{\sup _{1 \leq j \leq J_{0}}\left\|G^{(j)}\right\|_{\infty}<\varepsilon ; \sup _{\substack{1 \leq j \leq J \\ 1 \leq i \leq N \\ J_{0}<\ell \leq J}}\left(\left\|X^{(j)}\right\|_{\infty},\left\|Y_{i}^{(j)}\right\|_{\infty},\left\|G^{(\ell)}\right\|_{\infty}\right)>\varepsilon^{q}\right\} .
$$

Next notice that for any $j>J_{0}$, because $G^{(j)}(t)=\sum_{k=1}^{K} g_{k}(t) f_{k}(t),\left\|G^{(j)}\right\|_{\infty} \leq$ $g_{*} \sup _{k}\left\|f_{k}\right\|_{\infty}$ where the $f_{k}$ are from earlier in the ladder in the sense of (7.3). Hence for any $j$, we have that if

$$
\sup _{1 \leq i \leq N}\left(\left\|X^{(l)}\right\|_{\infty},\left\|Y_{i}^{(l)}\right\|_{\infty},\left\|G^{(l)}\right\|_{\infty}\right) \leq \varepsilon_{l+1} \text { for } l \leq j-1
$$

then $\left\|G^{(j)}\right\|_{\infty} \leq g_{*} \varepsilon_{j}<\varepsilon_{j}^{\frac{151}{152}}=\tilde{\varepsilon}_{j}$ for $\varepsilon$ sufficiently small. Restricting to $\varepsilon$ small enough so that $g_{*} \varepsilon_{j}<\varepsilon_{j}^{\frac{151}{152}}$ for all $1 \leq j \leq J$ implies that $A^{+}(j)=A(j)$ for all $1 \leq j \leq J$.

Next observe that $A(j) \cap B(j+1)^{c}=A(j+1)$ and hence $A^{+}(j) \cap B(j+1)^{c}=$ $A^{+}(j+1)$ for $\varepsilon$ sufficiently small. Iterating this observation, with the convention 
$A^{+}(0)=\Omega_{q *}(\mathscr{G}, \varepsilon) \cap \Omega_{q}(\mathscr{G}, \varepsilon)$, we obtain

$$
\begin{aligned}
A^{+}(0) & =\left[A^{+}(0) \cap B(1)^{c}\right] \cup\left[A^{+}(0) \cap B(1)\right] \\
& =A^{+}(1) \cup\left[A^{+}(0) \cap B(1)\right] \\
& =\left[A^{+}(1) \cap B(2)^{c}\right] \cup\left[A^{+}(1) \cap B(2)\right] \cup\left[A^{+}(0) \cap B(1)\right] \\
& =A^{+}(2) \cup\left[A^{+}(1) \cap B(2)\right] \cup\left[A^{+}(0) \cap B(1)\right] \\
& =A^{+}(J) \cup \bigcup_{j=0}^{J-1}\left[A^{+}(j) \cap B(j+1)\right] .
\end{aligned}
$$

Since $q$ was picked sufficiently small so that $\varepsilon_{J+1}<\varepsilon^{q}$, we observe that $A^{+}(J)$ is empty since on $A^{+}(J)$

$\sup _{\substack{1 \leq j \leq J \\ 1 \leq i \leq N \\ J_{0}<l \leq J}}\left(\left\|X^{(j)}\right\|_{\infty},\left\|Y_{i}^{(j)}\right\|_{\infty},\left\|G^{(l)}\right\|_{\infty}\right) \leq \varepsilon_{J+1}<\varepsilon^{q}<\sup _{\substack{1 \leq j \leq J \\ 1 \leq i \leq N \\ J_{0}<l \leq J}}\left(\left\|X^{(j)}\right\|_{\infty},\left\|Y_{i}^{(j)}\right\|_{\infty},\left\|G^{(l)}\right\|_{\infty}\right)$

which cannot be satisfied.

Recall that $\Omega_{\sharp}(\varepsilon)=\bigcup_{j=1}^{J} \Omega_{\sharp}^{\prime}\left(\tilde{\varepsilon}_{j}\right)$. Let $\Omega_{*}^{\prime}\left(H^{(j)}, \tilde{\varepsilon}_{j}\right)$ be the set defined bellow in Lemma 7.5. For all $q$ sufficiently small, $\Omega_{q *}(\mathscr{G}, \varepsilon) \subset \Omega_{*}^{\prime}\left(H^{(j)}, \tilde{\varepsilon}_{j}\right)$ for $j=$ $1, \cdots, J+1$. Decrease $q_{0}$ so this holds. With this choice Lemma 7.5 implies that $C(j) \subset \Omega_{\sharp}^{\prime}\left(\tilde{\varepsilon}_{j}\right)$. Since clearly $A^{+}(j) \cap B(j+1) \subset C(j+1)$, combining all of these observations produces

$$
\Omega_{q *}(\mathscr{G}, \varepsilon) \cap \Omega_{q}(\mathscr{G}, \varepsilon)=A^{+}(0) \subset \bigcup_{j=1}^{J} C(j) \subset \bigcup_{j=1}^{J} \Omega_{\sharp}^{\prime}\left(\tilde{\varepsilon}_{j}\right)=\Omega_{\sharp}(\varepsilon) .
$$

Lastly we give a version of the preceding proposition which begins with $L^{p}$ estimates in time on the $\left\{G^{(j)}: 1 \leq j \leq J_{0}\right\}$ rather than $L^{\infty}$ estimates.

Corollary 7.3. Fix $T>0$. For any $\ell>0$, define

$\Omega_{q, \ell}(\mathscr{G}, \varepsilon)=\left\{\sup _{1 \leq j \leq J_{0}} \int_{0}^{T}\left|G^{(j)}(s)\right|^{\ell} d s<\varepsilon ; \sup _{\substack{1 \leq j \leq J \\ 1 \leq i \leq N}}\left(\left\|X^{(j)}\right\|_{\infty},\left\|Y_{i}^{(j)}\right\|_{\infty},\left\|G^{(j)}\right\|_{\infty}\right)>\varepsilon^{q}\right\}$.

There exist positive constants $q=q(J, \ell)$ and $\varepsilon_{0}=\varepsilon_{0}(J, T, N, \ell)$ so that for all $\varepsilon \in$ $\left(0, \varepsilon_{0}\right]$

$$
\Omega_{q *}(\mathscr{G}, \varepsilon) \cap \Omega_{q, \ell}(\mathscr{G}, \varepsilon) \subset \Omega_{\sharp}(\varepsilon) .
$$

Proof. We begin by translating the bound

$$
\sup _{1 \leq j \leq J_{0}} \int_{0}^{T}\left|G^{(j)}(s)\right|^{\ell} d s<\varepsilon
$$

into a bound of the form $\sup _{1 \leq j \leq J_{0}}\left\|G^{(j)}\right\|_{\infty} \leq \varepsilon^{\beta}$ for some $\beta \in(0,1)$. 
Notice that

$$
\left|G^{(j)}(s)-G^{(j)}(r)\right|=\left|\int_{r}^{s} H^{(j)}(t) d t\right| \leq|s-r|\left\|H^{(j)}\right\|_{\infty} .
$$

Without loss of generality we assume $q<\frac{1}{150}$. Hence on $\Omega_{q *}(\mathscr{G}, \varepsilon) \cap\left\{\sup _{i}\left\|W_{i}\right\|_{\infty}>\varepsilon^{-\frac{1}{150}}\right\}^{c}$

$$
\left\|H^{(j)}\right\|_{\infty} \leq\left\|X^{(j)}\right\|_{\infty}+\sum_{i=1}^{N}\left\|Y_{i}^{(j)}\right\|_{\infty}\left\|W_{i}^{(j)}\right\|_{\infty} \leq \varepsilon^{-\frac{1}{150}}+N \varepsilon^{-\frac{1}{75}} \leq(N+1) \varepsilon^{-\frac{1}{75}}
$$

In other words, $\mathscr{H}_{1}(G) \leq(N+1) \varepsilon^{-\frac{1}{75}}$ on $\Omega_{q *}(\mathscr{G}, \varepsilon) \cap\left\{\sup _{i}\left\|W_{i}\right\|_{\infty}>\varepsilon^{-\frac{1}{150}}\right\}^{c}$. Then Lemma 7.6 below implies $\sup _{j \leq J_{0}}\left\|G^{(j)}\right\|_{\infty} \leq(2+N) \varepsilon^{\beta_{0}}<\varepsilon^{\beta_{1}}$ for $\varepsilon$ sufficiently small where $\beta_{0}=\frac{74}{75} \frac{1}{1+\ell}$ and $\beta_{1}=\frac{1}{2} \frac{1}{1+\ell}$. Now notice that $\Omega_{*}\left(\mathscr{G}, \varepsilon^{\beta_{1}}\right) \subset$ $\Omega_{*}(\mathscr{G}, \varepsilon)$ because $\beta_{1}<1$. Hence the result follows from Proposition 7.2 and the fact that $\left\{\sup _{i}\left\|W_{i}\right\|_{\infty}>\varepsilon^{-\frac{1}{150}}\right\} \subset \Omega_{\sharp}(\varepsilon)$.

\subsection{The Basic Estimates}

Let

$$
G(t)=G_{0}+\int_{0}^{t} H(s) d s
$$

Now let $H(s)$ be any stochastic process of the form

$$
H(s)=X(s)+\sum_{i=1}^{N} Y_{i}(s) W_{i}(s) \stackrel{\text { def }}{=} X(s)-Z(s)
$$

where $X(s)$, and $Y_{1}(s), \ldots, Y_{N}(s)$ are Lipschitz continuous stochastic processes and $\left\{W_{1}(s), \ldots, W_{N}(s)\right\}$ are mutually independent standard Wiener processes with $W_{i}(0)=$ $0,1 \leq i \leq N$.

Next given $\varepsilon>0$, define the following subsets of the probability space:

$$
\begin{aligned}
& \hat{\Omega}_{*}(H, \varepsilon)=\left\{\sup _{1 \leq i \leq N}\left(\left\|Y_{i}\right\|_{\infty}\right) \leq \varepsilon^{-\frac{1}{28}} ; \sup _{1 \leq i \leq N}\left(\mathscr{H}_{1}\left(Y_{i}\right), \mathscr{H}_{1}(X)\right) \leq \varepsilon^{-\frac{1}{28}}\right\}, \\
& \Omega_{*}^{\prime}(H, \varepsilon)=\left\{\sup _{1 \leq i \leq N}\left(\|X\|_{1},\left\|Y_{i}\right\|_{1}\right) \leq \varepsilon^{-\frac{1}{150}}\right\}
\end{aligned}
$$

Lemma 7.4. Let $\varepsilon>0$. Assume $\mathscr{H}_{\alpha}(H) \leq c \varepsilon^{-\gamma}$ for some fixed $\alpha>\gamma>0$. Then $\|G\|_{\infty} \leq \varepsilon$ implies $\|H\|_{\infty} \leq(2+c) \varepsilon^{\frac{\alpha-\gamma}{1+\alpha}}$.

Proof. For any $s \in[0, t]$, let $r_{1} \leq s \leq r_{2}$ such that $r_{2}-r_{1}=\varepsilon^{\frac{1+\gamma}{1+\alpha}}$. Notice that by the assumption $H(r) \geq H(s)-c \varepsilon^{-\gamma}\left|r_{2}-r_{1}\right|^{\alpha}$ for any $r \in\left[r_{1}, r_{2}\right]$. Hence we have

$$
2 \varepsilon \geq G\left(r_{2}\right)-G\left(r_{1}\right)=\int_{r_{1}}^{r_{2}} H(r) d r \geq\left|r_{2}-r_{1}\right|\left(H(s)-c \varepsilon^{-\gamma}\left|r_{2}-r_{1}\right|^{\alpha}\right) .
$$


Rearranging this gives, $H(s) \leq \frac{2 \varepsilon}{\left|r_{2}-r_{1}\right|}+c \varepsilon^{-\gamma}\left|r_{2}-r_{1}\right|^{\alpha}=(2+c) \varepsilon^{\frac{\alpha-\gamma}{1+\alpha}}$. The same argument from above gives a complementary lower bound and completes the result.

Next, we have the following result.

Lemma 7.5. There exists a $\varepsilon_{0}=\varepsilon_{0}(N)$ so that for every $\varepsilon \in\left(0, \varepsilon_{0}\right]$ and stochastic process $G(t)$, of the form given above, one has

$$
\Omega_{*}^{\prime}(H, \varepsilon) \cap\left\{\|G\|_{\infty}<\varepsilon ; \sup _{1 \leq i \leq N}\left(\|X\|_{\infty},\left\|Y_{i}\right\|_{\infty}\right)>\varepsilon^{\frac{1}{151}}\right\} \subset \Omega_{\sharp}^{\prime}(\varepsilon) .
$$

Proof. The result will follow from Lemma 7.9 of the next subsection after some ground work is laid. As before, set $\Delta=\varepsilon^{\frac{14}{75}}$ and recall that by definition $\Omega_{\sharp}^{\prime}(\varepsilon)=$ $\Omega_{a}(\Delta) \cup \Omega_{b}(\Delta) \cup \Omega_{c}(\Delta)$. Then notice that $\mathscr{H}_{\frac{1}{4}}(f) \leq \mathscr{H}_{1}(f), \mathscr{H}_{\frac{1}{4}}(f+g) \leq \mathscr{H}_{\frac{1}{4}}(f)+$ $\mathscr{H}_{\frac{1}{4}}(g)$, and $\mathscr{H}_{\frac{1}{4}}(f g) \leq \mathscr{H}_{\frac{1}{4}}(g)\|f\|_{\infty}+\mathscr{H}_{\frac{1}{4}}(f)\|g\|_{\infty}$ for all functions $f$ and $g$. Hence on $\Omega_{c}(\Delta)^{c} \cap \Omega_{*}^{\prime}(H, \varepsilon)$

$$
\begin{aligned}
\mathscr{H}_{\frac{1}{4}}(H) & \leq \mathscr{H}_{1}(X)+\sum_{i} \mathscr{H}_{1}\left(Y_{i}\right)\left\|W_{i}\right\|_{\infty}+\mathscr{H}_{\frac{1}{4}}\left(W_{i}\right)\left\|Y_{i}\right\|_{\infty} \\
& \leq \varepsilon^{-\frac{1}{150}}+2 N \varepsilon^{-\frac{1}{75}} \leq(1+2 N) \varepsilon^{-\frac{1}{75}}
\end{aligned}
$$

Hence by Lemma7.4 one has that on $\Omega_{c}(\Delta)^{c} \cap \Omega_{*}^{\prime}(H, \varepsilon),\|G\|_{\infty}<\varepsilon$ implies $\|H\|_{\infty} \leq$ $(1+2 N) \varepsilon^{\frac{71}{75}}<\Delta=\varepsilon^{\frac{14}{75}}$ for all $\varepsilon$ sufficiently small. Next observe that because $\Delta=\varepsilon^{\frac{14}{75}}, \Omega_{*}^{\prime}(H, \varepsilon) \subset \hat{\Omega}_{*}(H, \Delta)$ where $\hat{\Omega}_{*}(H, \Delta)$ is the set which was defined in equation (7.5). In light of this, Lemma 7.9 implies that

$$
\Omega_{*}^{\prime}(H, \varepsilon) \cap\left\{\|G\|_{\infty}<\varepsilon ; \sup _{i}\left\|Y_{i}\right\|_{\infty}>\varepsilon^{\frac{1}{75}}\right\} \subset \Omega_{\sharp}^{\prime}(\varepsilon) .
$$

Now on

$$
\Omega_{c}(\Delta)^{c} \cap \Omega_{*}^{\prime}(H, \varepsilon) \cap\left\{\|G\|_{\infty}<\varepsilon ; \sup _{i}\left\|Y_{i}\right\|_{\infty} \leq \varepsilon^{\frac{1}{75}}\right\}
$$

one has that $\|X\|_{\infty} \leq\|H\|_{\infty}+\sum\left\|Y_{i}\right\|_{\infty}\left\|W_{i}\right\|_{\infty} \leq \varepsilon^{\frac{14}{75}}+N \varepsilon^{\frac{1}{75}} \varepsilon^{-\frac{1}{150}}$ which is less than $\varepsilon^{\frac{1}{151}}$ for $\varepsilon$ sufficiently small. Hence

$$
\Omega_{c}(\Delta)^{c} \cap \Omega_{*}^{\prime}(H, \varepsilon) \cap\left\{\|G\|_{\infty}<\varepsilon ; \sup _{i}\left\|Y_{i}\right\|_{\infty} \leq \varepsilon^{\frac{1}{75}}\right\} \cap\left\{\|X\|_{\infty}>\varepsilon^{\frac{1}{151}}\right\}
$$

is empty. Combining this observation with 7.6 implies

$$
\Omega_{*}^{\prime}(H, \varepsilon) \cap\left\{\|G\|_{\infty}<\varepsilon\right\} \cap\left[\left\{\sup _{i}\left\|Y_{i}\right\|_{\infty}>\varepsilon^{\frac{1}{75}}\right\} \cup\left\{\|X\|_{\infty}>\varepsilon^{\frac{1}{151}}\right\}\right] \subset \Omega_{\sharp}^{\prime}(\varepsilon) .
$$

Since $\left\{\sup _{i}\left\|Y_{i}\right\|_{\infty}>\varepsilon^{\frac{1}{151}}\right\} \subset\left\{\sup _{i}\left\|Y_{i}\right\|_{\infty}>\varepsilon^{\frac{1}{75}}\right\}$, the proof is complete.

Lemma 7.6. For any $\varepsilon>0$ and $\ell>0, \int_{0}^{T}|G(s)|^{\ell} d s<\varepsilon$ and $\mathscr{H}_{\alpha}(G)<c \varepsilon^{-\gamma}$ implies $\|G\|_{\infty}<(1+c) \varepsilon^{\frac{\alpha-\gamma}{1+\ell \alpha}}$ 
Proof. By Chebyshev's inequality for any $\beta>0$, we have $\lambda\left\{x:|G(x)|>\varepsilon^{\beta}\right\} \leq$ $\varepsilon^{1-\ell \beta}$ where $\lambda$ is Lebesgue measure. Hence $\|G\|_{\infty} \leq \varepsilon^{\beta}+c \varepsilon^{(1-\ell \beta) \alpha} \varepsilon^{-\gamma}$. Setting $\beta=\frac{\alpha-\gamma}{1+\ell \alpha}$ proves the result.

\subsection{The Main Technical Estimate:}

Let $H(s)$ be as in 7.4 from the preceding section. Define the following piecewise constant approximation of the $Z$ from the definition of $H$ :

$$
Z^{*}(s)=-\sum_{i=1}^{N} Y_{i}^{*}(s) W_{i}(s)
$$

where $Y_{i}^{*}(s)=\sum_{k=1}^{m} \mathbf{1}_{I_{k}}(s) \sup _{s \in I_{k}} Y_{i}(s)$ and $\mathbf{1}_{I_{k}}$ is the indicator function of the set $I_{k}=\left[t_{k-1}, t_{k}\right)$.

For any $k$, and process $\zeta(s)$ defined on $\left[t_{k-1}, t_{k}\right]$, we define the $\delta$-scale quadratic variation on $\left[t_{k-1}, t_{k}\right]$ by

$$
Q_{k}(\zeta)=\sum_{\ell=1}^{M(k)}\left[\delta_{\ell}^{k} \zeta\right]^{2}
$$

Lemma 7.7. On $\hat{\Omega}_{*}(H, \Delta) \cap \Omega_{c}(\Delta)^{c}$ for any $k=1, \ldots, m$, we have the following estimates: $Q_{k}(X) \leq 2 \Delta^{\frac{109}{42}}, Q_{k}\left(Z^{*}\right) \leq 2 Q_{k}(Z)+4 N^{2} \Delta^{\frac{25}{21}}$. Moreover, on $\hat{\Omega}_{*}(H, \Delta) \cap$ $\Omega_{c}(\Delta)^{c} \cap\left\{\sup _{s \in I_{k}}|H(s)|<\Delta\right\}, Q_{k}(Z) \leq 2 Q_{k}(X)+8 \Delta^{\frac{4}{3}}+8 \Delta^{2}$ and $Q_{k}\left(Z^{*}\right) \leq(40+$ $\left.4 N^{2}\right) \Delta^{\frac{25}{21}}$.

Proof. For brevity, we suppress the $k$ dependence of $M(k)$ and $s_{\ell}(k)$. The first inequality follows from

$$
\begin{aligned}
Q_{k}(X) & =\sum_{\ell=1}^{M}\left[X\left(s_{\ell}\right)-X\left(s_{\ell-1}\right)\right]^{2} \\
& \leq \sum_{\ell=1}^{M}\left[\delta \Delta^{-\frac{1}{28}}\right]^{2} \leq M \delta^{2} \Delta^{-\frac{1}{14}}=\left(\Delta^{-\frac{2}{3}}+1\right) \Delta^{\frac{10}{3}} \Delta^{-\frac{1}{14}}=\Delta^{\frac{109}{42}}+\Delta^{\frac{139}{42}}<2 \Delta^{\frac{109}{42}}
\end{aligned}
$$

To see the second implication, first notice that

$$
\begin{aligned}
Q_{k}\left(Z^{*}\right) & \leq \sum_{\ell=1}^{M}\left(2\left[\left|Z^{*}\left(s_{\ell-1}\right)-Z\left(s_{\ell-1}\right)\right|+\left|Z^{*}\left(s_{\ell}\right)-Z\left(s_{\ell}\right)\right|\right]^{2}+2\left[Z\left(s_{\ell-1}\right)-Z\left(s_{\ell}\right)\right]^{2}\right) \\
& \leq 2 Q_{k}(Z)+4 \sum_{\ell=0}^{M-1}\left[Z^{*}\left(s_{\ell}\right)-Z\left(s_{\ell}\right)\right]^{2}+4 \sum_{\ell=1}^{M}\left[Z^{*}\left(s_{\ell}\right)-Z\left(s_{\ell}\right)\right]^{2} .
\end{aligned}
$$


Next note that

$$
\begin{aligned}
\sum_{\ell=1}^{M}\left[Z^{*}\left(s_{\ell}\right)-Z\left(s_{\ell}\right)\right]^{2} & \leq \sum_{\ell=1}^{M}\left[\sum_{i=1}^{N}\left[Y_{i}\left(s_{\ell}\right)-Y_{i}^{*}\right] W_{i}\left(s_{\ell}\right)\right]^{2} \\
& \leq \Delta^{-\frac{1}{7}} \frac{N^{2}}{\delta} \sum_{\ell=1}^{M}(\ell \delta)^{2} \delta \leq \frac{N^{2}}{3 \delta} \Delta^{\frac{20}{7}}=\frac{N^{2}}{3} \Delta^{\frac{25}{21}}
\end{aligned}
$$

and similarly $\sum_{\ell=0}^{M-1}\left[Z^{*}\left(s_{\ell}\right)-Z\left(s_{\ell}\right)\right]^{2} \leq \frac{N^{2}}{3} \Delta^{\frac{25}{21}}$. Combining this estimate with the previous gives the second result.

The third result follows from $H(s)=X(s)-Z(s)$ and

$$
\begin{aligned}
Q_{k}(Z) & =\sum_{\ell=1}^{M}\left[Z\left(s_{\ell}\right)-Z\left(s_{\ell-1}\right)\right]^{2} \\
& \leq \sum_{\ell=1}^{M} 2\left[\left|Z\left(s_{\ell}\right)-X\left(s_{\ell}\right)\right|+\left|Z\left(s_{\ell-1}\right)-X\left(s_{\ell-1}\right)\right|\right]^{2}+2\left[X\left(s_{\ell}\right)-X\left(s_{\ell-1}\right)\right]^{2} \\
& \leq 8 \Delta^{2} M+2 Q_{k}(X) \leq 8 \Delta^{2}\left(\frac{\Delta}{\delta}+1\right)+2 Q_{k}(X) .
\end{aligned}
$$

Lastly, combining the three previous estimates produces the final estimate.

To aid in the analysis of $Y^{*}$, consider a general process of the form

$$
\zeta(s)=\sum_{i=1}^{N} a_{i}(s) W_{i}(s)
$$

where the $W_{i}$ are independent standard Wiener processes and the $a_{i}(s)$ are constant on the intervals $I_{k}=[(k-1) \Delta, k \Delta)$ for each $k=1, \ldots, m$. As before, for $k=1, \ldots m$, we define

$$
Q_{k}(\zeta)=\sum_{\ell=1}^{M(k)}\left(\sum_{i=1}^{N} a_{i}\left(s_{\ell}(k)\right) \delta_{\ell}^{k} W_{i}\right)^{2}
$$

where $s_{\ell}(k)$ and $\delta_{\ell}^{k}$ are as defined at the start of Section 7.1 Notice that if we define

$$
U=\sum_{i=1}^{N} a_{i}^{2} \sum_{\ell=1}^{M(k)}\left(\hat{\boldsymbol{\delta}}_{\ell}^{k} W_{i}\right)^{2} \quad V=\sum_{\substack{(i, j) \in\{1, \ldots, N\}^{2} \\ i \neq j}} a_{i} a_{j} \sum_{\ell=1}^{M(k)}\left(\hat{\boldsymbol{\delta}}_{\ell}^{k} W_{i}\right)\left(\hat{\boldsymbol{\delta}}_{\ell}^{k} W_{j}\right),
$$

where $a_{i}=a_{i}((k+1) \Delta)$ and $\hat{\delta}_{\ell}^{k} f=\left(\delta_{\ell}^{k} f\right) / \sqrt{\delta_{\ell}^{k}}$ then $Q_{k}(\zeta)=\frac{\Delta}{M(k)}(U+V)$.

Lemma 7.8. For $\sigma>\frac{8}{7}, \Delta \in\left(0,6^{\frac{-7}{7 \sigma-8}}\right)$ and $k=1, \ldots, m$,

$$
\left\{Q_{k}(\zeta)<\Delta^{\sigma} ; \Delta^{\frac{1}{14}}<\sup _{i}\left|a_{i}\right| \leq \Delta^{-\frac{1}{28}}\right\} \subset \Omega_{a}(\Delta) \cup \Omega_{b}(\Delta)
$$


Proof. First notice that because $\frac{8}{7}<\sigma$ and $\Delta<6^{\frac{-7}{\sigma-8}}, \Delta^{\sigma}-\frac{1}{2} \Delta^{\frac{8}{7}}<-\frac{1}{3} \Delta^{\frac{8}{7}}$ so

$$
\begin{aligned}
& \left\{Q_{k}(\zeta)<\Delta^{\sigma} ; \Delta^{\frac{1}{14}}<\sup _{i}\left|a_{i}\right| \leq \Delta^{-\frac{1}{28}}\right\} \\
& \subset\left\{\frac{\Delta}{M(k)} U<\frac{1}{2} \Delta^{\frac{8}{7}} ; \sup _{i}\left|a_{i}\right|>\Delta^{\frac{1}{14}}\right\} \cup\left\{\frac{\Delta}{M(k)} V<\Delta^{\sigma}-\frac{1}{2} \Delta^{\frac{8}{7}} ; \sup _{i}\left|a_{i}\right|<\Delta^{-\frac{1}{28}}\right\} \\
& \subset\left\{\frac{\Delta}{M(k)} U<\frac{1}{2} \Delta^{\frac{8}{7}} ; \sup _{i}\left|a_{i}\right|>\Delta^{\frac{1}{14}}\right\} \cup\left\{\frac{\Delta}{M(k)}|V|>\frac{1}{3} \Delta^{\frac{8}{7}} ; \sup _{i}\left|a_{i}\right|<\Delta^{-\frac{1}{28}}\right\}
\end{aligned}
$$

Now

$$
\left\{U \leq \frac{1}{2} \Delta^{\frac{1}{7}} M(k) ; \sup _{i}\left|a_{i}\right|>\Delta^{\frac{1}{14}}\right\} \subset\left\{\inf _{i} \sum_{\ell=1}^{M(k)}\left(\hat{\delta}_{\ell}^{k} W_{i}\right)^{2} \leq \frac{1}{2} M(k)\right\} \subset \Omega_{a}(\Delta)
$$

and

$$
\begin{aligned}
&\{|V|>\left.\frac{1}{3} \Delta^{\frac{1}{7}} M(k) ; \sup _{i}\left|a_{i}\right|<\Delta^{-\frac{1}{28}}\right\} \\
& \subset\left\{\sup _{\substack{i, j) \\
i \neq j}}\left|a_{i}\right|\left|a_{j}\right|\left|\sum_{\ell=1}^{M(k)}\left(\hat{\delta}_{\ell}^{k} W_{i}\right)\left(\hat{\delta}_{\ell}^{k} W_{j}\right)\right|>\frac{\Delta^{\frac{1}{7}}}{3 N^{2}} M(k) ; \sup _{i}\left|a_{i}\right|<\Delta^{-\frac{1}{28}}\right\} \\
& \subset\left\{\sup _{\substack{i, j) \\
i \neq j}}\left|\sum_{\ell=1}^{M(k)}\left(\hat{\delta}_{\ell}^{k} W_{i}\right)\left(\hat{\delta}_{\ell}^{k} W_{j}\right)\right|>\frac{\Delta^{\frac{3}{14}}}{3 N^{2}} M(k)\right\} \subset \Omega_{b}(\Delta)
\end{aligned}
$$

The following result is the main result of this section.

Lemma 7.9. For all $\Delta \in\left(0,\left(40+4 N^{2}\right)^{-42}\right]$ and all stochastic processes $H(s)$ of the form (7.4)

$$
\hat{\Omega}_{*}(H, \Delta) \cap\left\{\|H\|_{\infty}<\Delta ; \sup _{i}\left\|Y_{i}\right\|_{\infty}>\Delta^{\frac{1}{14}}\right\} \subset \Omega_{a}(\Delta) \cup \Omega_{b}(\Delta) \cup \Omega_{c}(\Delta) .
$$

We will prove this result by showing that on $\hat{\Omega}_{*}(H, \Delta)$ as $\Delta \rightarrow 0$, if

$$
\sup _{s \in[0, T]}|H(s)|<\Delta \text { and } \sup _{i} \sup _{s \in[0, T]}\left|Y_{i}(s)\right|>\Delta^{\frac{1}{14}}
$$

then the approximate quadratic variation of the Wiener processes at the scale $\delta$ is abnormally small or $\sup _{i}\left\|W_{i}\right\|_{\infty}>\Delta^{-\frac{1}{28}}$.

Proof of Lemma 7.9 From the last estimate in Lemma7.7 we have that on $\hat{\Omega}_{*}(H, \Delta) \cap$ $\Omega_{c}(\Delta)^{c} \cap\left\{\sup _{s \in I_{k}}|H(s)|<\Delta\right\}, Q_{k}\left(Z^{*}\right) \leq\left(40+4 N^{2}\right) \Delta^{\frac{25}{21}}<\Delta^{\frac{49}{42}}$ for $\Delta \in(0,(40+$ 
$\left.\left.4 N^{2}\right)^{-42}\right]$. Here $Z^{*}$ is the approximation defined in (7.7). Now Lemma 7.8 with $\sigma=\frac{49}{42}\left(>\frac{8}{7}\right)$ implies that

$$
\Omega_{c}(\Delta)^{c} \cap \hat{\Omega}_{*}(H, \Delta) \cap\left\{\sup _{s \in I_{k}}|H(s)|<\Delta ; \sup _{i} \sup _{s \in I_{k}}\left|Y_{i}(s)\right|>\Delta^{\frac{1}{14}}\right\} \subset \Omega_{a}(\Delta) \cup \Omega_{b}(\Delta)
$$

for all $k=1, \ldots$ and $\Delta \in\left(0,\left(40+4 N^{2}\right)^{-42}\right]$.

Continuing, we have that

$$
\begin{aligned}
\Omega_{c}(\Delta)^{c} & \cap \hat{\Omega}_{*}(H, \Delta) \cap\left\{\|H\|_{\infty}<\Delta ; \sup _{i}\left\|Y_{i}\right\|_{\infty}>\Delta^{\frac{1}{14}}\right\} \\
& \subset \bigcup_{k=1}^{m}\left\{\sup _{s \in I_{k}}|H(s)|<\Delta ; \sup _{i} \sup _{s \in I_{k}}\left|Y_{i}(s)\right|>\Delta^{\frac{1}{14}}\right\} \cap \hat{\Omega}_{*}(H, \Delta) \cap \Omega_{c}(\Delta)^{c} \\
& \subset \Omega_{a}(\Delta) \cup \Omega_{b}(\Delta)
\end{aligned}
$$

\subsection{Estimates on the Size of $\Omega_{a}, \Omega_{b}$, and $\Omega_{c}$}

Since the events described by $\Omega_{a}$ and $\Omega_{b}$ are simply statements about collections of independent standard normal random variables, the following two estimates will give us the needed control.

Lemma 7.10. For $c \in(0,1)$ and $M>\frac{2}{1-c}$, setting $\gamma=c-1-\ln (c)>0$

$$
\begin{gathered}
\mathbb{P}\left(\sum_{\ell=1}^{M} \eta_{\ell}^{2} \leq c M\right) \leq \frac{1}{\sqrt{\pi M}} \exp \left(-\frac{1}{2} \gamma M\right) \\
\mathbb{P}\left(\left|\sum_{\ell=1}^{M} \eta_{\ell} \tilde{\eta}_{\ell}\right| \geq c M\right) \leq 2 \mathbb{P}\left(\sum_{\ell=1}^{M} \eta_{\ell} \tilde{\eta}_{\ell} \geq c M\right) \leq 2 \exp \left(-\frac{c^{2}}{4} M\right)
\end{gathered}
$$

where $\left\{\eta_{\ell}, \tilde{\eta}_{\ell}\right\}$ are a collection of $2 M$ mutually independent standard $N(0,1)$ random variables.

Proof. Notice that $\sum_{\ell=1}^{M} \eta_{\ell}^{2}$ is distributed as a $\chi^{2}$ random variable with $M$ degrees of freedom. Hence we have

$$
\mathbb{P}\left(\sum_{\ell=1}^{M} \eta_{\ell}^{2} \leq c M\right)=\frac{2^{-\frac{M}{2}}}{\Gamma\left(\frac{M}{2}\right)} \int_{0}^{c M} x^{\frac{M}{2}-1} e^{-\frac{x}{2}} d x
$$

Since $c<1$ and $M>\frac{2}{1-c}$, the integrand is bounded by $(c M)^{\frac{M}{2}-1} \exp \left(-c \frac{M}{2}\right)$. Combining this with $\Gamma\left(\frac{M}{2}\right) \geq \sqrt{\pi M}\left(\frac{M}{2 e}\right)^{\frac{M}{2}}$ implies that

$$
\begin{aligned}
\mathbb{P}\left(\sum_{\ell=1}^{M} \eta_{\ell}^{2} \leq c M\right) & \leq \frac{M^{-\frac{M}{2}} e^{\frac{M}{2}}}{\sqrt{\pi M}}(c M)^{\frac{M}{2}} \exp \left(-c \frac{M}{2}\right) \\
& \leq \frac{1}{\sqrt{\pi M}} \exp \left(\frac{M}{2}[-c+1+\ln (c)]\right) .
\end{aligned}
$$


Noticing that $-c+1+\ln (c)<0$ for $c \in(0,1)$ finishes the proof of the first statement.

For the second, note that for $\lambda \in(-1,1), \mathbb{E} \exp \left(\lambda \eta_{\ell} \tilde{\eta}_{\ell}\right)=\left(1-\lambda^{2}\right)^{-\frac{1}{2}}$. Hence for $\lambda \in\left[0, \frac{1}{2}\right]$,

$$
\begin{aligned}
\mathbb{P}\left(\sum_{\ell=1}^{M} \eta_{\ell} \tilde{\eta}_{\ell} \geq c M\right) & \leq \exp \left(-\lambda c M+\frac{1}{2}\left|\ln \left(1-\lambda^{2}\right)\right| M\right) \\
& \leq \exp \left(-\lambda c M+\lambda^{2} M\right)
\end{aligned}
$$

Taking $\lambda=\frac{c}{2}$ gives the result.

Corollary 7.11. For $\Delta \leq \frac{1}{2} \wedge T$,

$$
\begin{aligned}
& \mathbb{P}\left(\Omega_{a}(\Delta)\right) \leq \frac{2 T N}{\sqrt{\pi}} \Delta^{-\frac{2}{3}} \exp \left(-\frac{1}{20} \Delta^{-\frac{2}{3}}\right) \\
& \mathbb{P}\left(\Omega_{b}(\Delta)\right) \leq 6 N^{2} T \Delta^{-1} \exp \left(-\frac{1}{3 N^{2}} \Delta^{-\frac{19}{42}}\right) .
\end{aligned}
$$

In particular, if $\gamma=\min \left(\frac{1}{3 N^{2}}, \frac{1}{20}\right)$ then

$$
\mathbb{P}\left(\Omega_{a}(\Delta) \cup \Omega_{b}(\Delta)\right) \leq 8 T N^{2} \frac{\exp \left(-\gamma \Delta^{-\frac{2}{5}}\right)}{\Delta}
$$

Proof. First observe that the $\left\{\hat{\delta}_{\ell}^{k} W_{i}\right\}$ are independent $N(0,1)$ random variables. Since

$$
\Omega_{a} \subset \bigcup_{k=0}^{m} \bigcup_{i=1}^{N}\left\{\sum_{\ell=1}^{M(k)}\left(\hat{\delta}_{\ell}^{k} W_{i}\right)^{2} \leq \frac{M(k)}{2}\right\},
$$

$m \leq \frac{T}{\Delta}+1$ and $\Delta^{-\frac{2}{3}}-1 \leq M \leq \Delta^{-\frac{2}{3}}$ the first result follows from Lemma 7.10 and bounding the previous expression by the sum of the probability of the sets on the right handside.

Proceeding in a fashion similar to the first estimate, the second bound follows from

$$
\Omega_{b} \subset \bigcup_{k=0}^{m} \bigcup_{(i, j) \in\{1, \ldots, N\}^{2}}\left\{\left|\sum_{\ell=1}^{M(k)}\left(\hat{\delta}_{\ell}^{k} W_{i}\right)\left(\hat{\delta}_{\ell}^{k} W_{j}\right)\right| \geq \frac{\Delta^{\frac{3}{1}} M(k)}{3 N^{2}}\right\},
$$

Combining the first two estimates gives the last quoted result.

Lemma 7.12. For any $p \geq 1$ there exists a $c=c(p, N, T)$ so that $\mathbb{P}\left(\Omega_{c}(\varepsilon)\right) \leq c \varepsilon^{p}$.

Proof. It is enough to show that $\mathbb{E}\left\|W_{i}\right\|_{\infty}^{\gamma}<c(T, \gamma)$ and $\mathbb{E}\left[\mathscr{H}_{\frac{1}{4}}\left(W_{i}\right)^{\gamma}\right]<c(T, \gamma)$ for any $\gamma \geq 1$. The first follows from the Doob's inequalities for the continuous martingale $W_{i}(s)$. The finiteness of the moments of the modules continuity of Wiener processes is given by Theorem 2.1 (p. 26) and the observation at the top of p. 28 
both in [RY94]. Together these imply that $\mathbb{E}\left[\mathscr{H}_{\alpha}\left(W_{i}\right)^{\gamma}\right]<\infty$ for all $\gamma>0$ as long as $\alpha \in\left(0, \frac{1}{2}\right)$. Since $\alpha=\frac{1}{4}$ in our setting, the proof is complete.

\section{Strict Positivity of the Density}

We will now give conditions under which for any $t>0$ and some orthogonal projection $\Pi$ of $\mathbb{L}^{2}$ onto a finite dimensional subspace $S$, the density $p(t, x)$ of the law of $\Pi w(t)$ with respect to Lebesgue measure on $S$ satisfies

$$
p(t, x)>0 \text {, for all } x \in S \text {. }
$$

Our proof will make use of a criterion for strict positivity of the density of a random variable, which was first established in the case of finite dimensional diffusions by Ben Arous and Léandre [BAL91]. It was then extended to general random variables defined on Wiener space by Aida, Kusuoka and Stroock [AKS93]. We follow the presentation in Nualart [Nua98]. However, there is one major difference between our case and the classical situation treated in those references. As noted at the start of Section 2] our SPDE can be solved pathwise. This means that the Wiener process $W(t)=\left(W_{k}(t)\right)_{k \in \mathscr{Z}_{*}}$ can be replaced by a fixed trajectory in $\Omega_{[0, t]} \stackrel{\text { def }}{=} C\left([0, t] ; \mathbb{R}^{\mathscr{Z}_{*}}\right)$. Hence, we do not really need the notion of a skeleton. Because of this we can prove a result which is slightly more general than usual in that our controls need not belong to the Cameron Martin space. Let $Q \in \mathscr{L}\left(\mathbb{R}^{\mathscr{Z}_{*}} ; \mathbb{L}^{2}\right)$ be such that if $\left\{q_{k}, k \in \mathscr{Z}_{*}\right\}$ is a standard basis for $\mathbb{R}^{\mathscr{Z}_{*}}$, then $Q q_{k}=e_{k}$.

The main result of this section is the following:

Theorem 8.1. Assume that $S_{\infty}=\mathbb{L}^{2}$. Let $t>0$, and $\Pi$ be an orthogonal projection of $\mathbb{L}^{2}$ onto a finite dimensional subspace $S \subset S_{\infty}=\mathbb{L}^{2}$. Let $x \in S$ be such that for some $0<s<t$ and all $w \in \mathbb{L}^{2}$ there exists $H \in C\left([s, t] ; \mathbb{R}^{\mathscr{Z}_{*}}\right)$ such that the solution of

$$
\left\{\begin{array}{l}
\frac{\partial w^{H}}{\partial r}(r)+B\left(w^{H}(r), w^{H}(r)\right)=v \Delta w^{H}(r)+Q \frac{\partial H}{\partial r}(r), r>s \\
w^{H}(s)=w
\end{array}\right.
$$

satisfies $\Pi w^{H}(t)=x$. Then the density $p(t, \cdot)$ of the law of the random variable $\Pi w(t)$ satisfies $p(t, x)>0$.

Note that equation (8.2) makes perfect sense even when $H$ is not differentiable in time. To see this define $\bar{w}^{H}(s) \stackrel{\text { def }}{=} w^{H}(s)-Q H(s)$ and note that the equation for $\bar{w}^{H}(s)$ is a well known type of equation, to which existence and uniqueness results apply (cf. [CF88, FP67]).

Corollary 8.2. Let $\Pi$ be any orthogonal projection of $\mathbb{L}^{2}$ onto a finite dimensional space. If $S_{\infty}=\mathbb{L}^{2}$ then the density $p(t, \cdot)$ of the random variable $\Pi w(t)$ satisfies $p(t, x)>0$ for all $x \in \Pi \mathbb{L}^{2}$. 
Proof of Corollary 8.2 The results almost follows from the published version of Theorem 9 in Agrachev and Sarychev [AS03] which states that under some assumptions for any $s \in(0, t)$, the controllability assumption of Theorem 8.1 is satisfied with a control $H \in W^{1, \infty}\left(s, t ; \mathbb{R}^{\mathscr{Z}}\right)$. The increasing family of sets which describes the way the randomness spreads is slightly different the $S_{n}$. Furthermore, they do not state the result for arbitrary projection only the span of a finite number of Fourier modes. However, in private communications with the authors they have verified that the sets $S_{n}$ may be used and that an arbitrary finite dimensional projection may be taken. As an aside, Romito [Rom02] has proven this formulation of controllability of the Galerkin approximations under our assumptions.

Remark 8.3. It is worth pointing out that the exact control ability of the projections if far from the exact controllability in all of $\mathbb{L}^{2}$. In fact the later does not hold with smooth in space and $L^{2}$ in time controls. This would imply that the density was supported on $\mathbb{L}^{2}$ which is not true as its support is contained in functions which are analytic is space [Mat98 Mat02].

The rest of the section is devoted to the proof of Theorem 8.1 Our proof is based on the following result, which is a variant of Proposition 4.2.2 in Nualart [Nua98].

Proposition 8.4. Let $F \in C\left(\Omega_{[0, t]} ; S\right)$ where $S=\Pi \mathbb{L}^{2}$ is a finite dimensional vector space such that $H \mapsto F(H)$ is twice differentiable in the directions of $H^{1}\left(0, t ; \mathbb{R}^{\mathscr{Z}}\right)$, and these exist $D F(\cdot) \in C\left(\Omega_{[0, t]} ;\left[L^{2}(0, t ; S)\right]^{\mathscr{Z}_{*}}\right)$ and $D^{2} F(\cdot) \in C\left(\Omega_{[0, t]} ;\left[L^{2}\left((0, t)^{2} ; S\right)\right]^{\mathscr{Z}_{*} \times \mathscr{Z}_{*}}\right)$ such that for all $j, \ell \in \mathscr{Z}_{*}$ and $h, g \in L^{2}(0, t ; \mathbb{R})$,

$$
\begin{gathered}
\left.\frac{d}{d \varepsilon} F\left(H+\varepsilon \int_{0}^{\cdot} q_{j} h(s) d s\right)\right|_{\varepsilon=0}=\int_{0}^{t} D_{j, s} F(H) h(s) d s, \\
\left.\frac{d^{2}}{d \varepsilon d \delta} F\left(H+\varepsilon \int_{0}^{\cdot} q_{j} h(s) d s+\delta \int_{0} q_{\ell} g(s) d s\right)\right|_{\substack{\varepsilon=0 \\
\delta=0}}=\int_{0}^{t} \int_{0}^{t} D_{j, s ; \ell, r}^{2} F(H) h(s) g(r) d s d r .
\end{gathered}
$$

We assume moreover that

$$
H \mapsto\left(F(H), D F(H), D^{2} F(H)\right)
$$

is continuous and locally bounded from $\Omega_{[0, t]}$ into $S \times\left[L^{2}(0, t ; S)\right]^{\mathscr{Z}_{*}} \times\left[L^{2}\left([0, t]^{2} ; S\right)\right]^{\mathscr{Z}_{*} \times \mathscr{Z}_{*}}$, and that there exists $H \in \Omega_{[0, t]}$ such that $F(H)=x$ and

$$
\operatorname{det}\left(\sum_{k \in \mathscr{Z}_{*}} \int_{0}^{t} D_{k, s} F^{i}(H) D_{k, s} F^{j}(H) d s\right)>0 .
$$

Then, if the law of $F(W)$ has a density $p(t, \cdot), p(t, x)>0$.

Proof. Since the proof is almost identical to that of Proposition 4.2.2 in [Nua98], we only indicate the differences with the latter proof. 
In this proof, $H$ is one specific element of $\Omega_{[0, t]}$ satisfying $F(H)=x$ and (8.4). Let $h_{j}(s)=D_{., s} F^{j}(H), j \in \mathscr{Z}_{*}$. Clearly, $h_{j} \in L^{2}\left(0, t ; \mathbb{R}^{\mathscr{Z}}\right)$. For each $z \in \mathbb{R}^{\mathscr{Z}_{*}}$, let

$$
\left(T_{z} W\right)(t) \stackrel{\text { def }}{=} W(t)+\sum_{j \in \mathscr{Z}_{*}} z_{j} \int_{0}^{t} h_{j}(s) d s
$$

and

$$
g(z, W)=F\left(T_{z} W\right)-F(W) .
$$

It follows from our assumptions that for any $W \in \Omega_{[0, t]}, g(\cdot, W) \in C^{2}\left(B_{1}(0) ; \mathbb{R}^{\mathscr{Z}}\right)$, and for any $\beta>1$, there exists $C(\beta)$ such that

$$
\|W\|_{\infty, t} \leq C(\beta) \Rightarrow\|g(\cdot, W)\|_{C^{2}\left(B_{1}(0)\right)} \leq \beta
$$

where $\|W\|_{\infty, t} \stackrel{\text { def }}{=} \sup _{0 \leq s \leq t}|W(s)|$, and the notation $B_{\alpha}(0)$ stands for the open ball in $\mathbb{R}^{\mathscr{Z}}$ centered at 0 , with radius $\alpha$.

Assume for a moment that in addition

$$
\left|\operatorname{det} g^{\prime}(0)\right| \geq \frac{1}{\beta}
$$

It then follows from Lemma 4.2.1 in [Nua98] that there exists $c_{\beta} \in\left(0, \frac{1}{\beta}\right)$ and $\delta_{\beta}>0$ such that $g(\cdot, W)$ is diffeomorphic from $B_{c_{\beta}}(0)$ onto a neighborhood of $B_{\delta_{\beta}}(0)$.

We now define the random variable $\mathscr{H}_{\beta}$, which plays exactly the same role in the rest of our proof as $H_{\beta}$ in [Nua98], but is defined slightly differently. We let

$$
\mathscr{H}_{\beta}=k_{\beta}\left(\|W\|_{\infty, t}\right) \alpha_{\beta}\left(\left|\operatorname{det}\left\langle D F^{i}(W), D F^{j}(H)\right\rangle\right|\right),
$$

where $\langle\cdot, \cdot\rangle$ denotes the scalar product in $L^{2}\left(0, t ; \mathbb{R}^{\mathscr{Z}_{*}}\right) ; k_{\beta}, \alpha_{\beta} \in C(\mathbb{R} ;[0,1]), k_{\beta}(x)=$ 0 whenever $|x| \geq \beta, k_{\beta}(x)=1$ whenever $|x| \leq \beta-1 ; \alpha_{\beta}(x)=0$ whenever $|x| \leq$ $1 / \beta, \alpha_{\beta}(x)>0$ whenever $|x|>1 / \beta$, and $\alpha_{\beta}(x)=1$ if $|x| \geq 2 / \beta$.

The rest of the proof follows exactly the argument in [Nua98] pages 181 and 182. We only have to make explicit the sequence $T_{N}^{H}, N=1,2, \ldots$ of absolutely continuous transformations of $\Omega_{[0, t]}$ equipped with Wiener measure, which is used at the end of the proof. For $N=1,2, \ldots$, let $t_{i}^{N}=(i t) / N, 0 \leq i \leq N$. We define

$$
\left(T_{N}^{H} W\right)(t)=W(t)+\int_{0}^{t}\left(\dot{H}_{N}(s)-\dot{W}_{N}(s)\right) d s
$$

where

$$
\begin{aligned}
\dot{H}_{N}(s) & =\sum_{i=1}^{N-1} \frac{H\left(t_{i}^{N}\right)-H\left(t_{i-1}^{N}\right)}{t_{i}^{N}-t_{i-1}^{N}} \mathbf{1}_{\left[t_{i}^{N}, t_{i+1}^{N}\right)}(s), \\
\dot{W}_{N}(s) & =\sum_{i=1}^{N-1} \frac{W\left(t_{i}^{N}\right)-W\left(t_{i-1}^{N}\right)}{t_{i}^{N}-t_{i-1}^{N}} \mathbf{1}_{\left[t_{i}^{N}, t_{i+1}^{N}\right)}(s) .
\end{aligned}
$$

For any $W \in \Omega_{[0, t]}$, as $N \rightarrow \infty$,

$$
\sup _{0 \leq s \leq t}\left|\left(T_{N}^{H} W\right)(s)-H(s)\right| \rightarrow 0 .
$$


Hence, by the continuity of $F$ and $D F$, we also have

$$
\begin{aligned}
F\left(T_{N}^{H} W\right) & \rightarrow F(H) \\
D F\left(T_{N}^{H} W\right) & \rightarrow D F(H),
\end{aligned}
$$

and moreover

$$
\lim _{M \rightarrow \infty} \sup _{N} \mathbb{P}\left(\left\|T_{N}^{H} W\right\|_{\infty, t}>M\right)=0 .
$$

This provides exactly the version of $(\mathrm{H} 2)$ from Nualart, which is needed here to complete the proof.

All that remains is to prove the following lemma:

Proposition 8.5. Under the assumptions of Theorem 8.1] if $F=\Pi w(t)$, there exists $H \in \Omega_{[0, t]}$ such that $F(H) \stackrel{\text { def }}{=} \Pi w^{H}(t)=x$, and (8.4] holds.

Proof. Let $s \in(0, t)$ be the time which appears in the assumption of Theorem 8.1 Since $S_{\infty}=\mathbb{L}^{2}$, it follows from (3.2) that

$$
\mathbb{P}\left(\bigcap_{\phi \in \mathbb{H}^{1}, \phi \neq 0}\{\langle\mathscr{M}(s) \phi, \phi\rangle>0\}\right)=1 .
$$

We choose an arbitrary Brownian trajectory $W$ in this set of measure one. We then choose, according to the assumption of Theorem 8.1 an $H_{x} \in \Omega_{[0, t]}$ satisfying $H_{x}(s)=0$, such that the solution $\left\{w^{H_{x}}(r), s \leq r \leq t\right\}$ of 8.2 with initial condition $w^{H_{x}}(s)=w\left(s ; w_{0}, W\right)$, the value at time $s$ of the solution of 1.1 corresponding to the trajectory $W$ which we chose above, satisfies $\Pi w^{H_{x}}(t)=x$. It remains to show that the condition $(8.4)$ is satisfied, with $H \in \Omega_{[0, t]}$ defined by

$$
H(r)= \begin{cases}W(r), & \text { if } 0 \leq r \leq s \\ W(s)+H_{x}(r), & \text { if } s<r \leq t .\end{cases}
$$

We shall write

$$
w^{H}(r)= \begin{cases}w(r, W), & \text { if } 0 \leq r \leq s, \\ w^{H_{x}}(r), & \text { if } s<r \leq t .\end{cases}
$$

All we need to show is that for any fixed $\phi \in S, \phi \neq 0$,

$$
\sum_{k \in \mathscr{Z}_{*}} \int_{0}^{t}\left\langle D_{k, r} w^{H}(t), \phi\right\rangle^{2} d r>0
$$

Note that

$$
\begin{aligned}
\sum_{k \in \mathscr{Z}_{*}} \int_{0}^{t}\left\langle D_{k, r} w^{H}(t), \phi\right\rangle^{2} d r & \geq \sum_{k \in \mathscr{Z}_{*}} \int_{0}^{s}\left\langle D_{k, r} w^{H}(t), \phi\right\rangle^{2} d r \\
& =\sum_{k \in \mathscr{Z}_{*}} \int_{0}^{s}\left\langle D_{k, r} w^{H}(s), U^{t, \phi}(s)\right\rangle^{2} d s
\end{aligned}
$$


where $\left\{U^{t, \phi}(s), 0 \leq s \leq t\right\}$ solves the backward equation (2.5), associated to the corresponding $w$ trajectory, and the last identity follows from Proposition 2.3. In view of our specific choice of $W$, since from Lemma B.1 $U^{t, \phi}(s) \in \mathbb{H}^{1}$, it suffices to check that $U^{t, \phi}(s) \neq 0$. This follows from "backward uniqueness" (maybe we should say "forward uniqueness" since the $U^{t, \phi}$ equation is a backward equation!), see Theorem I.1 in Bardos, Tartar [BT73], whose assumptions are clearly verified by the $U^{t, \phi}(\cdot)$ equation (2.5). Specifically, since if $\phi=0$ then $U^{t, \phi}(s)=0$ for all $s \leq t$ one knows that no other terminal condition $\phi$ at time $t$ can lead to $U^{t, \phi}(s)=0$ for $s \leq t$.

\section{Conclusion}

We have proven under reasonable nondegeneracy conditions that the law of any finite dimensional projection of the solution of the stochastic Navier Stokes equation with additive noise possesses a smooth, strictly positive density with respect to Lebesgue measure. In particular, it was shown that four degrees of freedom are sufficient to guarantee nondegeneracy.

It is reasonable to ask if four is the minimal size set which produces finite dimensional projections with a smooth density. The nondegeneracy condition concentrates on the wave numbers were both the sin and cos are forced. Since this represents the translation invariant scales in the forcing, it is a mild restriction to require that whenever either of the sin or cos of a given wave number is forced, then both are forced. Under this assumption, forcing only two degrees of freedom corresponds to forcing both degrees of freedom associated to a single wave number $k$. It is easy to see that the subspace $\left\{u \in \mathbb{L}^{2}:\langle u, \sin (j \cdot x)\rangle=\langle u, \cos (j \cdot x)\rangle=\right.$ 0 for $j \neq k\}$ is invariant under the dynamics with such a forcing. Hence if the initial condition lies in this two dimensional subspace, the conclusions of Theorem 1.1] fail to hold. See [HM04] for a more complete discussion of this and other cases where the nondegeneracy condition fails.

We have concentrated on the 2D Navier Stokes equations forced by finite number of Wiener processes. However there are a number of ways one could extend these results. The choice of a forcing with finitely many modes was made for sim-

plicity in a number of technical lemmas, in particular in section 7.1 There appears to be no fundamental obstruction to extending the method to the cases with infinitely many forcing terms if the covariances satisfy an appropriate summability condition. In addition, the methods of this paper should equally apply to other polynomial nonlinearities, such as stochastic reaction diffusion equations with additive noise. In contrast, handling non-additive forcing in a nonlinear equation would require nontrivial extensions of the present work. Since in the linearization, stochastic integrals of nonadapted processes would appear, it is not certain that the line of argument in this paper would succeed. 
Acknowledgment. We would like to thank Andrei Agrachev, Gérard Ben Arous, Yuri Bakhtin, Weinan E, Thierry Gallouet, Martin Hairer, George Papanicolaou, and Yakov Sinai for stimulating and useful discussions. We also thank Martin Hairer who after reading a preliminary version of this paper suggested a better notation for the Fourier basis which we proceeded to adopt. We also thank the anonymous referee for her (or his) comments and corrections. JCM was partial supported by the Institute for Advanced Study and the NSF under grants DMS-9971087 and DMS-9729992. JCM would also like to thank the Université de Provence and the IUF which supported his stays in Marseilles during the summers of 2002-2004 when this work was undertaken.

\section{Appendix A: Estimates on the Enstrophy}

Define $\mathscr{E}_{0}=\sum_{k \in \mathscr{Z}_{*}}\left\|e_{k}\right\|^{2}$ where the $e_{k}$ were the functions used to define the forcing in (1.3). In general we define the $\alpha$-th spatial moment of the forcing to be $\mathscr{E}_{\alpha}=\sum_{k \in \mathscr{Z}_{*}}|k|^{2 \alpha}\left\|e_{k}\right\|^{2}$. With this notation, we have the following estimate.

Lemma A.1. Given any $\varepsilon \in(0,1)$, there exists a $\gamma=\gamma(\varepsilon)$ such that

$$
\mathbb{P}\left\{\sup _{s \in[0, t]}\|w(s)\|^{2}+2 \varepsilon v \int_{0}^{s}\|w(r)\|_{1}^{2} d r-\mathscr{E}_{0} s>\|w(0)\|^{2}+K\right\}<e^{-\gamma K}
$$

for all $K \geq 0$.

Proof. The Lemma follows from the exponential martingale estimate after one notices that the quadratic variation of the martingale in the equation for the enstrophy is controlled by $\int_{0}^{t}\|w(r)\|_{1}^{2} d r$. See [Mat02b] Lemma A.2 or [Mat02b] Lemma A.1 for the details and related lemmas in exactly this setting or Lemma A.3 below for a similar argument.

From the previous result, we obtain the following.

Corollary A.2. There exists a constant $\eta_{0}=\eta_{0}(T, v)>0$ so that for any $\eta \in$ $\left(0, \eta_{0}\right]$ there exists a constant $c=c(T, \eta, v)$ so that

$$
\mathbb{E} \exp \left(\eta \sup _{0 \leq s \leq T}\|w(s)\|^{2}\right) \leq c \mathbb{E} \exp \left(\eta\|w(0)\|^{2}\right)
$$

and

$$
\mathbb{E} \exp \left(v \eta \int_{0}^{T}\|w(s)\|_{1}^{2} d s\right) \leq c \mathbb{E} \exp \left(\eta\|w(0)\|^{2}\right)
$$

We will also need the following result which gives quantitative estimates on the regularization of the $\mathbb{H}^{1}$-norm.

Lemma A.3. Given a time $T>0$ and $p \geq 0$, there exists positive constants $c=$ $c\left(v, T, p, \mathscr{E}_{1}\right)$ so that

$$
\mathbb{E} \sup _{0 \leq s \leq T}\left[\|w(s)\|^{2}+s v\|w(s)\|_{1}^{2}\right]^{p} \leq c\left[1+\|w(0)\|^{4 p}\right]
$$


Proof. Defining $\zeta(s)=\|w(s)\|^{2}+s v\|w(s)\|_{1}^{2}$, we have for all $s \in[0, T]$

$$
\begin{aligned}
\zeta_{s}-\mathscr{E}_{0} s-\frac{1}{2} \mathscr{E}_{1} s^{2} & =\|w(0)\|^{2}+\int_{0}^{s} 2 r v\left[-v\|w(r)\|_{2}^{2}+\langle B(w(r), w(r)), \Delta w(r)\rangle\right] d r \\
& -v \int_{0}^{s}\|w(r)\|_{1}^{2} d r+\sum_{k \in \mathscr{Z}_{*}} \int_{0}^{s} 2\left(1+r v|k|^{2}\right)\left\langle w(r), e_{k}\right\rangle d W_{k}(r) .
\end{aligned}
$$

Since

$$
|\langle B(w, w), \Delta w\rangle| \leq c\|w\|_{\frac{1}{2}}\|w\|_{1}\|w\|_{2} \leq v\|w\|_{2}^{2}+c(v)\|w\|^{4}
$$

one has

$$
\sup _{0 \leq s \leq T} \zeta_{s} \leq c\left(1+T^{2}\right)+c T^{2} \sup _{0 \leq s \leq T}\|w(s)\|^{4}+\sup _{0 \leq s \leq T} N_{s}
$$

where

$$
N_{s}=-v \int_{0}^{s}\left[\|w(r)\|^{2}+r\|w(r)\|_{2}^{2}\right] d r+M_{s}
$$

and

$$
M_{s}=\sum_{k \in \mathscr{Z}_{*}} \int_{0}^{s} 2\left(1+r v|k|^{2}\right)\left\langle w(r), e_{k}\right\rangle d W_{k}(r) .
$$

Notice that for all $s \in[0, T]$ and $\alpha>0$ sufficiently small $N_{s} \leq M_{s}-\frac{\alpha}{2}[M, M]_{s}$, where $[M, M]_{s}$ is the quadratic variation of the martingale $M_{s}$. Hence the exponential martingale estimate implies $\mathbb{P}\left(\sup _{s \leq T} N_{s}>\beta\right) \leq \mathbb{P}\left(\sup _{s \leq T} M_{s}-\frac{\alpha}{2}[M, M]_{s}>\beta\right) \leq$ $\exp (-\alpha \beta)$. This implies that the last term in $\mathrm{A} .1$ can be bounded by a constant depending only on $\alpha, T, v$ and the power $p$. By Corollary A.2 the third term in A.1 can be bounded by a constant which depends on the initial condition as stated as well as $\alpha, T, v$ and the power $p$.

\section{Appendix B: Estimates on the Linearization and its Adjoint}

Define the action of linearized operator $J_{s, t}$ on a $\phi \in \mathbb{L}^{2}$ by

$$
\left\{\begin{array}{l}
\frac{\partial}{\partial t} J_{s, t} \phi=v \Delta J_{s, t} \phi+B\left(w(t), J_{s, t} \phi\right)+B\left(J_{s, t} \phi, w(t)\right) \quad 0 \leq s \leq t ; \\
J_{s, s} \phi=\phi
\end{array}\right.
$$

and its time reversed, $\mathbb{L}^{2}$-adjoint $\bar{J}_{s, t}^{*}$ acting on $\phi \in \mathbb{L}^{2}$ by

$$
\left\{\begin{array}{l}
\frac{\partial}{\partial s} \bar{J}_{s, t}^{*} \phi+v \Delta \bar{J}_{s, t}^{*} \phi+B\left(w(s), \bar{J}_{s, t}^{*} \phi\right)-C\left(\bar{J}_{s, t}^{*} \phi, w(s)\right)=0 \quad 0 \leq s \leq t \\
\bar{J}_{t, t}^{*} \phi=\phi
\end{array}\right.
$$

If we define the operator $Q: \mathbb{R}^{\mathscr{Z}_{*}} \rightarrow \mathbb{L}^{2}$ by $\left(x_{k}\right)_{k \in \mathscr{Z}_{*}} \mapsto \sum x_{k} e_{k}$, then $V_{k, s}(t)=$ $J_{s, t} Q q_{k}$ were $\left\{q_{k}: k \in \mathscr{Z}_{*}\right\}$ is the standard basis for $\mathbb{R}^{\mathscr{Z}_{*}}$ and $U^{t, \phi}(s)=\bar{J}_{s, t}^{*} \phi$. Similarly for $h \in L_{l o c}^{2}\left(\mathbb{R}_{+}^{\mathscr{Z}_{*}}\right), D_{h} w(t)=\int_{0}^{t} J_{s, t} Q h(s) d s$. 
Lemma B.1. For any $T_{0}>0, \eta>0$ and $\alpha \in\{0,1\}$ there exists constants $\gamma=$ $\gamma\left(v, \eta, T_{0}^{\alpha}\right)$ and $c=c\left(v, T_{0}^{\alpha}, \eta\right)$ so that for all $\phi \in \mathbb{L}^{2}$ and $T \leq T_{0}$

$$
\begin{aligned}
& \sup _{0 \leq s \leq t \leq T}\left\|J_{s, t} \phi\right\|^{2}+\gamma(t-s)^{\alpha}\left\|J_{s, t} \phi\right\|_{1}^{2} \leq \exp \left(\eta \int_{0}^{T}\|w(r)\|_{1}^{2} d r+c T\right)\left(\|\phi\|^{2}+(1-\alpha)\|\phi\|_{1}^{2}\right) \\
& \sup _{0 \leq s \leq t \leq T}\left\|\bar{J}_{s, t}^{*} \phi\right\|^{2}+\gamma(t-s)^{\alpha}\left\|\bar{J}_{s, t}^{*} \phi\right\|_{1}^{2} \leq \exp \left(\eta \int_{0}^{T}\|w(r)\|_{1}^{2} d r+c T\right)\left(\|\phi\|^{2}+(1-\alpha)\|\phi\|_{1}^{2}\right)
\end{aligned}
$$

where on the right hand side $(1-\alpha)\|\phi\|_{1}^{2}=0$ when $\alpha=1$ by convention for all $\phi$ even if $\|\phi\|_{1}=\infty$.

Proof. We start by deriving a number of bounds on the nonlinear terms. Using Lemma D.1 and standard interpolation inequalities produces for any $\delta>0$ and $\eta>0$ and some $c$,

$$
\begin{aligned}
2|\langle B(\phi, w), \phi\rangle| & \leq c\|\phi\|^{\frac{3}{2}}\|\phi\|_{1}^{\frac{1}{2}}\|w\|_{1} \leq \delta\|\phi\|_{1}^{2}+\|\phi\|^{2}\left(\eta\|w\|_{1}^{2}+\frac{c}{\delta \eta^{2}}\right) \\
2|\langle B(\phi, w), \Delta \phi\rangle| & \leq c\|w\|_{1}\|\phi\|_{1}^{\frac{1}{2}}\|\phi\|^{\frac{1}{2}}\|\phi\|_{2} \leq \delta\|\phi\|_{2}^{2}+\eta\|w\|_{1}^{2}\|\phi\|_{1}^{2}+\frac{c}{\eta \delta^{2}}\|w\|_{1}^{2}\|\phi\|^{2} \\
2|\langle B(w, \phi), \Delta \phi\rangle| & \leq c\|w\|_{1}\|\phi\|_{1}\|\phi\|^{\frac{1}{4}}\|\phi\|_{2}^{\frac{3}{4}} \leq \frac{c}{(\eta \delta)^{\frac{3}{2}}}\|w\|_{1}\|\phi\|_{1}\|\phi\|+(\eta \delta)^{\frac{1}{2}}\|w\|_{1}\|\phi\|_{1}\|\phi\|_{2} \\
& \leq \delta\|\phi\|_{1}^{2}+\frac{c}{\eta^{3} \delta^{4}}\|w\|_{1}^{2}\|\phi\|^{2}+\frac{\delta}{2}\|\phi\|_{2}^{2}+\eta\|w\|_{1}^{2}\|\phi\|_{1}^{2} \\
2|\langle B(\Delta \phi, w), \phi\rangle| & \leq c\|\phi\|_{1+\varepsilon}\|\phi\|_{1-\varepsilon}\|w\|_{1} \leq c\|w\|_{1}\|\phi\|\|\phi\|_{2} \leq \delta\|\phi\|_{2}^{2}+\frac{c}{\delta}\|w\|_{1}^{2}\|\phi\|^{2}
\end{aligned}
$$

We begin with bounding $J$ expression with $\alpha=0$. Setting $\zeta_{r}=\left\|J_{s, s+r} \phi\right\|^{2}+$ $\gamma\left\|J_{s, s+r} \phi\right\|_{1}^{2}, w_{r}=w(s+r)$ and $J_{r}=J_{s, s+r} \phi$ and using the above estimates with appropriately chosen constants produces

$$
\begin{aligned}
\frac{\partial \zeta_{r}}{\partial r}= & \frac{\partial}{\partial r}\left\|J_{r}\right\|^{2}+\gamma \frac{\partial}{\partial r}\left\|J_{r}\right\|_{1}^{2} \\
= & -2 v\left\|J_{r} \phi\right\|_{1}^{2}+2\left\langle B\left(J_{r}, w\right), J_{r}\right\rangle \\
& \quad+2 \gamma\left[-v\left\|J_{r}\right\|_{2}^{2}+\left\langle B\left(J_{r}, w\right), \Delta J_{r}\right\rangle+\left\langle B\left(w, J_{r}\right), \Delta J_{r}\right\rangle\right] \\
\leq & \left(\frac{c}{\eta^{2} v}+\frac{\eta}{2}\left\|w_{r}\right\|_{1}^{2}+\gamma c\left(\frac{1}{\eta v^{2}}+\frac{1}{\eta^{3} v^{4}}\right)\left\|w_{r}\right\|_{1}^{2}\right)\left\|J_{r}\right\|^{2}+\gamma \eta\left\|w_{r}\right\|_{1}^{2}\left\|J_{r}\right\|_{1}^{2}
\end{aligned}
$$

Hence for $\gamma$ sufficiently small there exist a constant $c$ so for all $r>0$

$$
\frac{\partial \zeta_{r}}{\partial r} \leq\left[\frac{c}{\eta^{2} v}+\eta\left\|w_{r}\right\|_{1}^{2}\right] \zeta_{r}
$$

which proves the first result for $J_{s, t}$. The result for $\alpha=1$ is identical except that we take $\zeta_{r}=\left\|J_{s, s+r} \phi\right\|^{2}+\gamma r\left\|J_{s, s+r} \phi\right\|_{1}^{2}$ and hence there is an extra term from the differentiating the coefficient of $\left\|J_{s, s+r} \phi\right\|_{1}^{2}$ and the fact that the resulting constants 
depend on the time interval $[0, T]$ over which $r$ ranges. See the proof of Lemma A.3

Turning to $\bar{J}_{s, t}^{*}$, we set $\bar{\zeta}_{r}=\left\|\bar{J}_{t-r, t}^{*} \phi\right\|^{2}+\gamma\left\|\bar{J}_{t-r, t}^{*} \phi\right\|^{2}, \bar{w}_{r}=w(t-r)$ and $\bar{J}_{r}^{*}=$ $\bar{J}_{t-r, t}^{*} \phi$ for all $r \in[0, t]$. The bar is to remind us that the process is time reversed. The argument proceeds as in the previous case. Again using the estimates above, we obtain

$$
\frac{\partial \bar{\zeta}_{r}}{\partial r} \leq\left(\frac{c}{v\left(1+\eta^{2}\right)}+\left(\frac{\eta}{2}+\frac{\gamma c}{v}\right)\left\|w_{r}\right\|_{1}^{2}\right)\left\|\bar{J}_{r}^{*}\right\|^{2}+\gamma \eta\left\|w_{r}\right\|_{1}^{2}\left\|\bar{J}_{r}^{*}\right\|_{1}^{2} .
$$

Hence for $\gamma$ small enough and $r \in[0, t]$,

$$
\frac{\partial \bar{\zeta}_{r}}{\partial r} \leq\left[\frac{c}{v\left(1+\eta^{2}\right)}+\eta\left\|w_{r}\right\|_{1}^{2}\right] \bar{\zeta}_{r}
$$

which proves the result.

\section{Appendix C: Higher Malliavin Derivatives of $w(t)$}

For notational brevity define $\tilde{B}(f, g)=B(f, g)+B(g, f)$ for $f, g \in \mathbb{L}^{2}$. For $k \in$ $\mathscr{Z}_{*}$, we define $J_{s, t}^{(1)} e_{k}=J_{s, t} e_{k}$. For $n>1$ define $J_{s, t}^{(n)}$ acting on $\phi=\left(e_{k_{1}}, \ldots, e_{k_{n}}\right)$ with $k_{1}, \ldots, k_{n} \in \mathscr{Z}_{*}$ and with time parameters $s=\left(s_{1}, \ldots, s_{n}\right) \in \mathbb{R}_{+}^{n}$ by the equations

$$
\begin{aligned}
\frac{\partial}{\partial t} J_{s ; t}^{(n)} \phi & =v \Delta J_{s ; t}^{(n)} \phi+\tilde{B}\left(w(t), J_{s ; t}^{(n)} \phi\right)+F_{s ; t}^{(n)} \phi ; \quad t>\vee s \\
J_{s ; t}^{(n)} & =0 ; \quad t \leq \vee s
\end{aligned}
$$

where $\vee s=s_{1} \vee \cdots \vee s_{n}$. The operator $F_{s, t}^{(n)}$ applied to $\phi$ is defined by

$$
F_{s ; t}^{(n)} \phi=\sum_{(\alpha, \beta) \in \operatorname{part}(n)} \tilde{B}\left(J_{s_{\alpha} ; t}^{(|\alpha|)} \phi_{\alpha}, J_{s_{\beta} ; t}^{(|\beta|)} \phi_{\beta}\right)
$$

Here $\operatorname{part}(n)$ is the set of partitions of $\{1, \ldots, n\}$ into two sets, neither of them empty. $|\alpha|$ is the number of elements in $\alpha, \phi_{\alpha}=\left(\phi_{\alpha_{1}}, \ldots, \phi_{\alpha_{|\alpha|}}\right)$ and $s_{\alpha}=\left(s_{\alpha_{1}}, \ldots, s_{\alpha_{|\alpha|} \mid}\right)$. The partition $(\alpha, \beta)$ and $(\beta, \alpha)$ are viewed as the same partition.

First observe that when $n=1$, Lemma B.1 says that for any $\eta>0$ there is a $c=c(T, \eta)$ so for all $\phi \in \mathbb{R}^{\mathscr{Z}_{*}}$

$$
\sup _{0 \leq s \leq t \leq T}\left\|J_{s ; t}^{(1)} e_{k}\right\|_{1} \leq c \exp \left(\eta \int_{0}^{T}\|w(r)\|_{1}^{2} d r\right) \leq c \exp \left(\eta \int_{0}^{T}\|w(r)\|_{1}^{2} d r\right) .
$$

For $n>1$ with again $\phi=\left(e_{k_{1}}, \ldots, e_{k_{n}}\right)$ and $s \in \mathbb{R}_{+}^{n}$, we have the following estimate on $F^{(n)}$

$$
\left\|F_{s ; t}^{(n)} \phi\right\| \leq \sum_{(\alpha, \beta) \in \operatorname{part}(n)}\left\|\tilde{B}\left(J_{s_{\alpha} ; t}^{(|\alpha|)} \phi_{\alpha}, J_{s_{\beta} ; t}^{(|\beta|)} \phi_{\beta}\right)\right\| \leq c \sum_{(\alpha, \beta) \in \operatorname{part}(n)}\left\|J_{s_{\alpha} ; t}^{(|\alpha|)} \phi_{\alpha}\right\|_{1}\left\|J_{s_{\beta} ; t}^{(|\beta|)} \phi_{\beta}\right\|_{1} .
$$


Then the variation of constants formula implies that $J_{s ; t}^{(n)} \phi=\int_{V s}^{t} J_{r, t} F_{s ; r}^{(n)} \phi d r$ and hence

$$
\begin{aligned}
\left\|J_{s, t}^{(n)} \phi\right\|_{1} & \leq \int_{\vee s}^{t}\left\|J_{r, t} F_{s ; r}^{(n)} \phi\right\|_{1} d r \\
& \leq c \exp \left(\eta \int_{0}^{T}\|w(r)\|_{1}^{2} d r\right)\left(\int_{\vee s}^{t} \frac{1}{(r-\vee s)^{\frac{1}{2}}} d r\right) \sup _{\substack{\tau=\left(\tau_{1}, \cdots, \tau_{n}\right) \\
0 \leq \tau_{i} \leq t \\
\vee \tau \leq r \leq t}}\left\|F_{\tau ; r}^{(n)} \phi\right\| \\
& \leq c \exp \left(\eta \int_{0}^{T}\|w(r)\|_{1}^{2} d r\right) \sum_{(\alpha, \beta) \in \text { part }(n)} \sup _{\tau, r}\left\|J_{\tau_{\alpha} ; r}^{(|\alpha|)} \phi_{\alpha}\right\|_{1} \sup _{\tau, r}\left\|J_{\tau_{\beta} ; r}^{(|\beta|)} \phi_{\beta}\right\|_{1}
\end{aligned}
$$

Proceeding inductively, one obtains for any $\eta>0$ the existence for a $c=c(T, \eta, n)$ and $\gamma=\gamma(n)$ so that

$$
\left\|J_{s ; t}^{(n)} \phi\right\|_{1} \leq \int_{s}^{t}\left\|J_{r, t} F_{s, r} \phi\right\|_{1} d r \leq c \exp \left(\gamma \eta \int_{0}^{T}\|w(r)\|_{1}^{2} d r\right) .
$$

Now with $\phi$ and $s$ as above,

$$
D_{s_{1}, k_{1} ; \ldots ; s_{n}, k_{n}}^{n} w(t)=J_{s, t}^{(n)} \phi,
$$

and since $\eta$ was a arbitrary positive constant, by redefining it, one obtains that for any $\eta>0$, there exits a $c=c(T, \eta, n)$ so that

$$
\sup _{t \in(0, T]}\left\|D_{s_{1}, k_{1} ; \ldots ; s_{n}, k_{n}}^{n} w(t)\right\|_{1} \leq c \exp \left(\eta \int_{0}^{T}\|w(r)\|_{1}^{2} d r\right) .
$$

Combining this estimate with Lemma A.2 we obtain the following result. Letting $\mathbb{D}^{\infty}\left(\mathbb{H}_{1}\right)$ is the space of random variable taking values in $\mathbb{H}_{1}$ which are inifintely differentiable in the Malliavin sense and such that those derivatives have all moments finite, we have that: (see [Nua95] page 62 for the definition of $\mathbb{D}^{\infty}$ )

Lemma C.1. For any $\eta>0, t>0, p \geq 1$ and $n \geq 1$ there exists a constant $c=$ $c(t, v, \eta, p, n)$ so that

$\mathbb{E}\left[\left(\sum_{k_{1}, \ldots, k_{n} \in \mathscr{Z}_{*}} \int_{0}^{t} \cdots \int_{0}^{t}\left\|D_{s_{1}, k_{1} ; \ldots ; s_{n}, k_{n}}^{n} w(t)\right\|_{1}^{2} d s_{1} \cdots d s_{n}\right)^{p / 2}\right] \leq c \exp \left(\eta\|w(0)\|^{2}\right)$.

Hence $w(t) \in \mathbb{D}^{\infty}\left(\mathbb{H}_{1}\right)$ for all $t>0$.

\section{Appendix D: Estimates on the Nonlinearity}

In the following, Lemma we collect a few standard estimates on the nonlinearity and derive a few consequences from them. 
Lemma D.1. Let $\alpha_{i} \geq 0$ and either $\alpha_{1}+\alpha_{2}+\alpha_{3}>1$ or both $\alpha_{1}+\alpha_{2}+\alpha_{3}=1$ and $\alpha_{i} \neq 1$ for any $i$. Then the following estimates hold of all $f, g, h \in \mathbb{L}^{2}$ if the right hand side is well defined.

$$
|\langle B(f, g), h\rangle| \leq c\|f\|_{\alpha_{1}-1}\|g\|_{\alpha_{2}+1}\|h\|_{\alpha_{3}}
$$

In addition we have the following estimate. For any $\varepsilon>0$ there exists a $c=c(\varepsilon)$ with

$$
|\langle\nabla B(f, g), \nabla g\rangle| \leq c\|f\|_{1}\|g\|_{1}\|g\|_{1+\varepsilon}
$$

Proof. For the first result see Proposition 6.1 of [CF88] and recall that our $B$ is slightly different than theirs and that $\|\mathscr{K} f\|_{1}=\|f\|_{0}$. After translation, the result follows. For the second result we need to rearrange things. Setting $u=\left(u_{1}, u_{2}\right)=$ $\mathscr{K} f$ we have

$$
|\langle\nabla B(f, g), \nabla g\rangle|=\left|\int_{\mathbf{T}^{2}} \nabla[(u \cdot \nabla) g] \cdot \nabla g d x\right|
$$

Observe that $\nabla[(u \cdot \nabla) g] \cdot \nabla g=[(u \cdot \nabla) \nabla g] \cdot \nabla g+(\nabla u \nabla g) \cdot \nabla g$. Because $\nabla \cdot u=0$, $[(u \cdot \nabla) \nabla g] \cdot \nabla g=\frac{1}{2} \sum_{i} \nabla \cdot\left(u\left(\frac{\partial g}{\partial x_{i}}\right)^{2}\right)$. Hence, the integral of the first term is zero by stokes theorem and the fact that we are on the torus.

The integral of the second term over the domain is made of a finite number of terms of the form $\int \frac{\partial u_{j}}{\partial x_{i}} \frac{\partial g}{\partial x_{j}} \frac{\partial g}{\partial x_{i}} d x$. This term is dominated by $\left|\frac{\partial u_{j}}{\partial x_{i}}\right|_{L^{r}}\left|\frac{\partial g}{\partial x_{j}}\right|_{L^{p}}\left|\frac{\partial g}{\partial x_{i}}\right|_{L^{q}}$ for any $r, p, q>1$ with $\frac{1}{r}+\frac{1}{q}+\frac{1}{p}=1$. Recall that in two dimensions, the Sobolev space $W^{1,2}$ is embedded in $L^{r}$ for any $r<\infty$. Hence by taking $p=\frac{1}{2}, q>2$ sufficiently close to 2 and $r$ correspondingly large, we obtain the bound $c\left\|\frac{\partial u}{\partial x_{i}}\right\|\left\|_{1}\right\| \frac{\partial g}{\partial x_{j}}\|\| \frac{\partial g}{\partial x_{i}} \|_{\varepsilon}$ for any $\varepsilon>0$ and some $c=c(\varepsilon)$. The estimate is in turn bounded by $c\|f\|_{1}\|g\|_{1}\|g\|_{1+\varepsilon}$.

\section{Appendix E: Lipschitz and Supremum Estimates}

Let $S$ be a subspace of $\mathbb{L}^{2}$ spanned by a finite number of $\cos (x \cdot k)$ and $\sin (x \cdot k)$. Let $\Pi$ be the orthogonal projection onto $S$. Also let $\Pi_{0}$ be the orthogonal projection onto the directions directly forced by Wiener processes as defined in Proposition 3.7

Recall from Section 7 the definitions of $\mathscr{H}_{\alpha,[a, b]}(f),\|f\|_{\infty},\|f\|_{\infty,[a, b]}$ applied to functions of time taking values in $\mathbb{L}^{2}$.

For $0 \leq s<t \leq T$ one has

$$
\Pi_{0}^{\perp} w(t)=\Pi_{0}^{\perp} e^{v \Delta(t-s)} w(s)-\Pi_{0}^{\perp} \int_{s}^{t} e^{v \Delta(t-r)} B(w(r), w(r)) d r .
$$


Since $\int f(x) d x=\int g(x) d x=0,\|B(f, g)\| \leq c\|\nabla f\|\|\nabla g\|$ and $\left\|\left(e^{v \Delta(t-s)}-1\right) f\right\| \leq$ $\left(1-e^{-v \lambda(t-s)}\right)\|f\|$ for some fixed $\lambda>0$, one has

$$
\begin{aligned}
\left\|\Pi_{0}^{\perp}[w(t)-w(s)]\right\| & \leq 2\left[1-e^{-\lambda v(t-s)}\right]\|w(s)\|+2 \sup _{0 \leq r \leq T}\|\nabla w(r)\|^{2}|t-s| \\
& \leq c|t-s|\left[1+\|\nabla w\|_{\infty}^{2}\right] .
\end{aligned}
$$

Similarly,

$$
\begin{aligned}
\left\|U^{T, \phi}(t)-U^{T, \phi}(s)\right\| & \leq c|t-s|\left\|U^{T, \phi}(t)\right\|+c\|\nabla w\|_{\infty}\left\|\nabla U^{T, \phi}\right\|_{\infty}|t-s| \\
& \leq c\left[1+\|\nabla w\|_{\infty}^{2}+\left\|\nabla U^{T, \phi}\right\|_{\infty}^{2}\right]|t-s| .
\end{aligned}
$$

Also if

$$
R(t)=\Pi_{0} w(0)+\int_{0}^{t} v \Delta \Pi_{0} w(r)-\Pi_{0} B(w(r), w(r)) d r
$$

then

$$
\|R(t)-R(s)\| \leq c \int_{s}^{t}\|w(r)\| d r+c \int_{s}^{t}\|w(r)\|^{2} d r \leq c\left[1+\|w\|_{\infty}^{2}\right]|t-s|
$$

Next observe that $\|\Pi B(f, g)\| \leq c\|f\|\|g\|$ and $\|\Pi C(f, g)\| \leq c[\|f\|\|\nabla g\| \wedge\|g\|\|\nabla f\|]$.

Thus

$$
\begin{aligned}
\|\Pi C(f(t), g(t))-\Pi C(f(s), g(s))\| & \leq\|\Pi C(f(t)-f(s), g(t))\|+\|\Pi C(f(s), g(t)-g(s))\| \\
& \leq c\left[\|\nabla g\|_{\infty}\|f(t)-f(s)\|+\|\nabla f\|_{\infty}\|g(t)-g(s)\|\right]
\end{aligned}
$$

and

$$
\|\Pi B(f(t), g(t))-\Pi B(f(s), g(s))\| \leq c\left[\|\| g\left\|_{\infty}\right\| f(t)-f(s)\|+\| f\left\|_{\infty}\right\| g(t)-g(s) \|\right]
$$

We combine these observations in the following Lemma.

Lemma E.1. Let $\Pi$ and $\Pi_{0}$ be as above. In the notation of (7.1) and (7.2),

$$
\begin{aligned}
\mathscr{H}_{1}\left(\Pi_{0}^{\perp} w\right) & \leq c\left[1+\|\nabla w\|_{\infty}^{2}\right] \\
\mathscr{H}_{1}\left(U^{T, \phi}\right) & \leq c\left[1+\|\nabla w\|_{\infty}^{2}+\left\|\nabla U^{T, \phi}\right\|_{\infty}^{2}\right] \\
\mathscr{H}_{1}(R) & \leq c\left[1+\|w\|_{\infty}^{2}\right] \\
\mathscr{H}_{1}(\Pi B(f, g)) & \leq c\left[\|f\|_{\infty} \mathscr{H}_{1}(g)+\|g\|_{\infty} \mathscr{H}_{1}(f)\right] \\
\mathscr{H}_{1}(\Pi C(f, g)) & \leq c\left[\|\nabla f\|_{\infty} \mathscr{H}_{1}(g)+\|\nabla g\|_{\infty} \mathscr{H}_{1}(f)\right]
\end{aligned}
$$

for all $f, g \in \mathbb{L}^{2}$ smooth enough that each term on the right handside is finite.

Lastly we specialize these estimates to the setting of Proposition 3.7. Let $X^{\phi}$, $R$, and $Y_{k}^{\phi}$ be as defined in Proposition 3.7 for $w(t)$ and $U^{T, \phi}$ on the interval $[0, T]$. Define $\chi^{\phi}=\Pi X^{\phi}$ and $\Upsilon_{k}^{\phi}=\Pi Y_{k}^{\phi}$. We wish to obtain control of the Lipschitz constants over an interval $[t, T]$ with $t \in(0, T)$. 
Using the estimates from LemmaE.1 and the fact that $\|\nabla R\|_{\infty,[t, T]} \leq c\|R\|_{\infty,[t, T]} \leq$ $c\left(1+\|w\|_{\infty,[t, T]}^{2}\right)$, we obtain

$$
\begin{gathered}
\mathscr{H}_{1,[t, T]}\left(\chi^{\phi}\right) \leq c\left[\mathscr{H}_{1,[t, T]}\left(U^{T, \phi}\right)+\|\nabla w\|_{\infty,[t, T]} \mathscr{H}_{1,[t, T]}\left(U^{T, \phi}\right)+\left\|\nabla U^{T, \phi}\right\|_{\infty,[t, T]} \mathscr{H}_{1,[t, T]}\left(\Pi_{0}^{\perp} w\right)\right. \\
\left.+\|\nabla R\|_{\infty,[t, T]} \mathscr{H}_{1,[t, T]}\left(U^{T, \phi}\right)+\left\|\nabla U^{T, \phi}\right\|_{\infty,[t, T]} \mathscr{H}_{1,[t, T]}(R)\right] \\
\leq c\left[1+\|\nabla w\|_{\infty,[t, T]}^{4}+\left\|\nabla U^{T, \phi}\right\|_{\infty,[t, T]}^{4}\right]
\end{gathered}
$$

and

$$
\begin{aligned}
\mathscr{H}_{1,[t, T]}\left(\Upsilon_{k}^{\phi}\right) & \leq c\left[\mathscr{H}_{1,[t, T]}\left(U^{T, \phi}\right)+\left\|U^{T, \phi}\right\|_{\infty,[t, T]}^{2}\right] \\
& \leq c\left[1+\|\nabla w\|_{\infty,[t, T]}^{2}+\left\|\nabla U^{T, \phi}\right\|_{\infty,[t, T]}^{2}\right] .
\end{aligned}
$$

Similarly we have

$$
\begin{aligned}
\left\|\chi^{\phi}\right\|_{\infty,[t, T]} & \leq c\left[\left\|U^{T, \phi}\right\|_{\infty,[t, T]}+\left\|\nabla U^{T, \phi}\right\|_{\infty,[t, T]}\|\nabla w\|_{\infty,[t, T]}+\left\|\nabla U^{T, \phi}\right\|_{\infty,[t, T]}\|R\|_{\infty,[t, T]}\right] \\
& \leq c\left[1+\left\|\nabla U^{T, \phi}\right\|_{\infty,[t, T]}^{2}+\|\nabla w\|_{\infty,[t, T]}^{2}\right]
\end{aligned}
$$

and $\left\|\Upsilon_{k}^{\phi}\right\|_{\infty,[t, T]} \leq c\left\|U^{T, \phi}\right\|_{\infty,[t, T]}$

Recall that $\|f\|_{\alpha,[t, T]}=\max \left(\|f\|_{\infty,[t, T]}, \mathscr{H}_{\alpha,[t, T]}(f)\right)$. Combining the above estimates with Lemma B.1 produces

$$
\begin{aligned}
\left\|\chi^{\phi}\right\|_{1,[t, T]},\left\|\mathrm{\Upsilon}_{k}^{\phi}\right\|_{1,[t, T]} & \leq c\left[1+\left\|\nabla U^{T, \phi}\right\|_{\infty,[t, T]}^{4}+\|\nabla w\|_{\infty,[t, T]}^{4}\right] \\
& \leq c\left[1+\|\nabla w\|_{\infty,[t, T]}^{4}+\exp \left(\eta \int_{0}^{T}\|w(r)\|_{1}^{2} d r\right)\right]
\end{aligned}
$$

for all indices $i$, and $\phi$ with $\|\nabla \phi\| \leq M$. Here $\eta>0$ is arbitrary but $c$ depends on the choice of $\eta$ and $M$. In light of Corollary A.2 and Lemma A.3 which control the right hand side, we obtain the following result.

Lemma E.2. Given an $M>0$ define $S(M)=\{\phi:\|\nabla \phi\| \leq M\}$. Then for any $T>0$, $t \in(0, T), p \geq 1$, and $\eta>0$ there exists a positive constant $c=c(\eta, p, t, v, \mathscr{E} 1, M, T)$ such that

$$
\mathbb{E}\left(\sup _{\phi \in S(M)}\left[\left\|\chi^{\phi}\right\|_{1,[t, T]}^{p}+\sup _{k \in \mathscr{Z}_{*}}\left\|\mathrm{\Upsilon}_{k}^{\phi}\right\|_{1,[t, T]}^{p}\right]\right) \leq c \exp \left(\eta\|w(0)\|^{2}\right)
$$

\section{Bibliography}

[AKS93] S. Aida, S. Kusuoka, and D. Stroock. On the support of Wiener functionals. In Asymptotic problems in probability theory: Wiener functionals and asymptotics (Sanda/Kyoto, 1990), volume 284 of Pitman Res. Notes Math. Ser., pages 3-34. Longman Sci. Tech., Harlow, 1993.

[AS03] Andrei Agrachev and Andrey Sarychev. Navier-Stokes equations: controllability by means of low modes forcing. J. Math. Fluid Mech., 7(1):108-152, 2005.

[BAL91] G. Ben Arous and R. Léandre. Décroissance exponentielle du noyau de la chaleur sur la diagonale. II. Probab. Theory Related Fields, 90(3):377-402, 1991.

[BKL01] J. Bricmont, A. Kupiainen, and R. Lefevere. Ergodicity of the 2D Navier-Stokes equations with random forcing. Comm. Math. Phys., 224(1):65-81, 2001. Dedicated to Joel L. Lebowitz. 
[BT73] C. Bardos and L. Tartar. Sur l'unicité rétrograde des équations paraboliques et quelques questions voisines. Arch. Rational Mech. Anal., 50:10-25, 1973.

[CF88] Peter Constantin and Ciprian Foiaş. Navier-Stokes Equations. University of Chicago Press, Chicago, 1988.

[DL88] Robert Dautray and Jacques-Louis Lions. Analyse mathématique et calcul numérique pour les sciences et les techniques. Masson, Paris, 1988.

[DPZ96] Giuseppe Da Prato and Jerzy Zabczyk. Ergodicity for Infinite Dimensional Systems. Cambridge, 1996.

[EH01] J.-P. Eckmann and M. Hairer. Uniqueness of the invariant measure for a stochastic PDE driven by degenerate noise. Comm. Math. Phys., 219(3):523-565, 2001

[EM01] Weinan E and Jonathan C. Mattingly. Ergodicity for the Navier-Stokes equation with degenerate random forcing: finite-dimensional approximation. Comm. Pure Appl. Math., 54(11):1386-1402, 2001.

[EMS01] Weinan E, J. C. Mattingly, and Ya G. Sinai. Gibbsian dynamics and ergodicity for the stochastic forced navier-stokes equation. Comm. Math. Phys., 224(1), 2001.

[Fla94] Franco Flandoli. Dissipativity and invariant measures for stochastic Navier-Stokes equations. NoDEA, 1:403-426, 1994.

[FP67] C. Foiaş and G. Prodi. Sur le comportement global des solutions non-stationnaires des équations de Navier-Stokes en dimension 2. Rend. Sem. Mat. Univ. Padova, 39:1-34, 1967.

[HM04] Martin Hairer and Jonathan C. Mattingly. Ergodicity of the degenerate stochastic 2D Navier-Stokes equation. To appear in Annals of Mathematics.

[HS81] R. Holley and D. Stroock. Diffusions on an infinite-dimensional torus. J. Funct. Anal., 42(1):29-63, 1981.

[KS00] Sergei Kuksin and Armen Shirikyan. Stochastic dissipative PDEs and Gibbs measures. Comm. Math. Phys., 213(2):291-330, 2000.

[Mat98] Jonathan C. Mattingly. The Stochastically forced Navier-Stokes equations: energy estimates and phase space contraction. $\mathrm{PhD}$ thesis, Princeton University, 1998.

[Mat02] Jonathan C. Mattingly. The dissipative scale of the stochastics Navier-Stokes equation: regularization and analyticity. J. Statist. Phys., 108(5-6):1157-1179, 2002.

[Mat02b] Jonathan C. Mattingly. Exponential convergence for the stochastically forced NavierStokes equations and other partially dissipative dynamics. Comm. Math. Phys., 230(3):421462, 2002.

[Mat03] Jonathan C. Mattingly. On recent progress for the stochastic Navier Stokes equations. In Journées Équations aux dérivées partielles, Forges-les-Eaux, 2003. see http://arxiv.org/abs/math.PR/0409194 or http://www.math.sciences.univ-nantes.fr/edpa/2003/html/

[MB02] Andrew J. Majda and Andrea L. Bertozzi. Vorticity and incompressible flow, volume 27 of Cambridge Texts in Applied Mathematics. Cambridge University Press, Cambridge, 2002.

[MR04] R. Mikulevicius and B. L. Rozovskii. Stochastic Navier-Stokes equations for turbulent flows. SIAM J. Math. Anal. 35(5): 1250-1310, 2004. (electronic).

[MS99] J. C. Mattingly and Ya. G. Sinai. An elementary proof of the existence and uniqueness theorem for the Navier-Stokes equations. Commun. Contemp. Math., 1(4):497-516, 1999.

[Nor86] James Norris. Simplified Malliavin calculus. In Séminaire de Probabilités, XX, 1984/85, pages 101-130. Springer, Berlin, 1986.

[NP88] D. Nualart and E. Pardoux. Stochastic calculus with anticipating integrands. Probab. Th. Rel. Fields, 78:535-581, 1988.

[Nua95] David Nualart. The Malliavin calculus and related topics. Probability and its Applications. Springer-Verlag, New York, 1995. 
[Nua98] David Nualart. Analysis on Wiener space and anticipating stochastic calculus. In Lectures on probability theory and statistics (Saint-Flour, 1995), volume 1690 of Lecture Notes in Math., pages 123-227. Springer, Berlin, 1998.

[Oco88] Daniel Ocone. Stochastic calculus of variations for stochastic partial differential equations. J. Funct. Anal., 79(2):288-331, 1988.

[Rom02] Marco Romito. Ergodicity of the finite dimensional approximation of the 3D NavierStokes equations forced by a degenerate noise. J. Statist. Phys. 114, no. 1-2, (2004), 155-177.

[RY94] Daniel Revuz and Marc Yor. Continuous martingales and Brownian motion, volume 293 of Grundlehren der Mathematischen Wissenschaften. Springer-Verlag, Berlin, second edition, 1994.

[Str83] D. W. Stroock. Some applications of stochastic calculus to partial differential equations. In Ecole d'Été de Probabilités de Saint Flour, volume 976 of Lecture notes in Mathematics, pages 267-382. Springer, 1983.

Received June 2004.

Revised September 2005. 